QUANTUM DYNAMICS

BANACH CENTER PUBLICATIONS, VOLUME 120
INSTITUTE OF MATHEMATICS
POLISH ACADEMY OF SCIENCES
WARSZAWA 2020

\title{
STANDARD HOMOGENEOUS C*-ALGEBRAS AS COMPACT QUANTUM METRIC SPACES
}

\author{
KONRAD AGUILAR \\ Department of Mathematics and Computer Science (IMADA) \\ University of Southern Denmark, Campusvej 55, DK-5230 Odense M, Denmark \\ ORCID:0000-0003-2766-4694_E-mail: konrad.aguilar@asu.edu \\ TRISTAN BICE \\ Matematický Ústav $A V \check{C} R$ \\ Žitná 25, 11567 Praha 1, Czech Republic \\ ORCID: 0000-0003-1744-3943 E-mail: tristan.bice@gmail.com
}

\begin{abstract}
Given a compact metric space $X$ and a unital $\mathrm{C}^{*}$-algebra $A$, we introduce a family of seminorms on the $\mathrm{C}^{*}$-algebra of continuous functions from $X$ to $A$, denoted by $C(X, A)$, induced by classical Lipschitz seminorms that produce compact quantum metrics in the sense of Rieffel if and only if $A$ is finite-dimensional. As a consequence, we are able to isometrically embed $X$ into the state space of $C(X, A)$. Furthermore, we are able to extend convergence of compact metric spaces in the Gromov-Hausdorff distance to convergence of spaces of matrices over continuous functions on the associated compact metric spaces in Latrémolière's GromovHausdorff propinquity.
\end{abstract}

\section{Contents}

1. Introduction and background $\ldots \ldots \ldots \ldots \ldots$. . . . . . . . . . . . . . . . . . . .

2. Quantum metrics on standard homogeneous $\mathrm{C}^{*}$-algebras . . . . . . . . . . . . . . 86

3. Isometries from metrics into quantum metrics . . . . . . . . . . . . . . . . . . . 96

4. Convergence of standard homogeneous $\mathrm{C}^{*}$-algebras and finite-dimensional approxima-

tions . . . . . . . . . . . . . . . . . . . . . . . . . 102

References ...................................109

2010 Mathematics Subject Classification: Primary: 46L89, 46L30, 58B34.

Key words and phrases: noncommutative metric geometry, Monge-Kantorovich distance, quantum metric spaces, Lip-norms, circle algebras, homogeneous $\mathrm{C}^{*}$-algebras.

The paper is in final form and no version of it will be published elsewhere. 
1. Introduction and background. Compact quantum metric spaces introduced by Rieffel [27, 28, 30] (motivated by the work of Connes [8,9]) provide a framework for the study of noncommutative metric geometry just as spectral triples provide for noncommutative differential geometry and $\mathrm{C}^{*}$-algebras provide for noncommutative topology. These spaces are defined using a $\mathrm{C}^{*}$-algebra equipped with a seminorm that serves as noncommutative analogues to the Lipschitz seminorm on the $\mathrm{C}^{*}$-algebra of complex-valued continuous functions on a compact metric space. In this article, we will provide families of quantum metrics for the $\mathrm{C}^{*}$-algebra of continuous functions from a compact metric space to a finite-dimensional $\mathrm{C}^{*}$-algebra. As an application, we show that convergence of compact metric spaces in the Gromov-Hausdorff distance can be extended to convergence of spaces of matrices over continuous functions on the associated compact metric spaces in Latrémolière's quantum Gromov-Hausdorff propinquity. The canonical mapping $(X, \mathrm{~d}) \mapsto\left(C(X), \mathrm{L}_{\mathrm{d}}\right)$ from compact metric spaces to compact quantum metric spaces is continuous, and in fact a homeomorphism onto its image, from the Gromov-Hausdorff distance to Rieffel's quantum distance [29, Proposition 4.7] (this has been known since 2000 and also is true for Latrémolière's quantum Gromov-Hausdorff propinquity 20, Theorem 6.6]); this paper proves that $(X, \mathrm{~d}) \mapsto(C(X, \mathfrak{A}), \mathrm{L})$ remains continuous for the same topologies, when $\mathfrak{A}$ is a fixed finite-dimensional $\mathrm{C}^{*}$-algebra and $\mathrm{L}$ is introduced in this paper.

We note of another motivation for our work in this article. The quantum metrics we introduced will in particular endow standard circle algebras with quantum metrics, where we define a standard circle algebra to be $C(\mathbb{T}, \mathfrak{A})$, where $\mathbb{T}$ is the circle and $\mathfrak{A}$ is a finite-dimensional $\mathrm{C}^{*}$-algebra. Thus, this article is partly motivated by work of the first author and F. Latrémolière $\sqrt{1}-3$, in which they brought AF algebras into the realm on noncommutative metric geometry by, in part, finding various quantum metrics for $\mathrm{AF}$ algebras. Therefore, following the Elliott classification program [13], a next natural step is to develop quantum metrics for limit circle algebras or AT-algebras or inductive limits of circle algebras [11, page 159]. But, as done in [3], it is quite beneficial to first place quantum metrics on the $\mathrm{C}^{*}$-algebras of the inductive sequence that build the inductive limit. Thus, this article serves to provide a natural family of quantum metrics for circle algebras as a vital step for this pursuit.

Our construction will be based on the quantum metric induced by the Lipschitz seminorm on the $\mathrm{C}^{*}$-algebra of continuous functions on a compact metric space $\left(X, \mathrm{~d}_{X}\right)$, denoted by $C(X)$. Indeed, it is a well-known result (likely due to L. Kantorovich and see 12 for a reference) that the metric on the state space $\mathscr{S}(C(X))$ defined by

$$
\mathrm{mk}_{\mathrm{L}_{\mathrm{d}_{X}}}:(\mu, \nu) \in \mathscr{S}(C(X)) \times \mathscr{S}(C(X)) \longmapsto \sup _{\mathrm{L}_{\mathrm{d}_{X}}(f) \leqslant 1}|\mu(f)-\nu(f)|
$$

metrizes the weak* topology of $\mathscr{S}(C(X))$, the state space of $C(X)$, and

$$
\mathrm{L}_{\mathrm{d}_{X}}(f)=\sup _{x, y \in X, x \neq y} \frac{|f(x)-f(y)|}{\mathrm{d}_{X}(x, y)}
$$

is the Lipschitz constant of $f$, which we call the Lipschitz seminorm. Furthermore, the map

$$
\Delta: x \in\left(X, \mathrm{~d}_{X}\right) \longmapsto \delta_{x} \in\left(\mathscr{S}(C(X)), \mathrm{mk}_{\mathrm{L}_{\mathrm{d}_{X}}}\right)
$$


is an isometry onto its image, where $\delta_{x}: f \in C(X) \longmapsto f(x) \in \mathbb{C}$ is the Dirac point mass at $x$. Of course, even when $X$ is only compact Hausdorff, this map is a homeomorphism onto its image. Thus, the contribution of $m k_{L_{d_{X}}}$ is that this homeomorphism is strengthened to an isometry in the case when $X$ is compact metric.

The generalization of the Lipschitz seminorm to metric-space valued functions, and in particular, $\mathrm{C}^{*}$-algebra valued functions, is very natural. This paper studies these generalizations from the perspective of noncommutative metric geometry. First, in Section 2 the family of seminorms we develop on $C(X, \mathfrak{A})$ will produce quantum metrics if and only if $\mathfrak{A}$ is finite-dimensional. Next, for a particular choice of seminorm on $C(X, \mathfrak{A})$, the above $C(X)$ structure will be recovered in the unital $\mathrm{C}^{*}$-subalgebra of scalar-valued functions denoted by $C\left(X, \mathbb{C} 1_{\mathfrak{A}}\right)$ where $1_{\mathfrak{A}}$ is the identity of $\mathfrak{A}$ (also in Section 2 ). In Section 3 , an appropriate analogue to the above map, $\Delta$, will capture the metric structure of $X$ in the state space via the quantum metric by way of a bi-Lipschitz map for any of the seminorms we produce, and for particular natural choices of seminorms, the map will be an isometry just as $\Delta$ is in the classical case, and these cases will still be independent of $\mathfrak{A}$ and $X$. Furthermore, although some of our main results rest on the finite-dimensionality of $\mathfrak{A}$, we note that we are able to prove many crucial algebraic and analytic properties of our seminorms without the assumption of finite-dimensionality on $\mathfrak{A}$. And, finally, in Section 4 . we extend the convergence of compact metric spaces to a purely noncommutative setting by way of matrices over spaces of continuous functions.

Now, we provide some necessary background for the results of this paper.

Notation 1.1. Let $\mathfrak{A}$ be a unital $C^{*}$-algebra. Denote the $\mathrm{C}^{*}$-norm of $\mathfrak{A}$ by $\|\cdot\|_{\mathfrak{A}}$ and the unit of $\mathfrak{A}$ by $1_{\mathfrak{A}}$. If $\mathfrak{B}$ is a $\mathrm{C}^{*}$-subalgebra of $\mathfrak{A}$, then we use $\|\cdot\|_{\mathfrak{A} / \mathfrak{B}}$ to denote the quotient norm on $\mathfrak{A} / \mathfrak{B}$ induced by the $\mathrm{C}^{*}$-norm of $\mathfrak{A}$.

Denote the set of self-adjoint elements of $\mathfrak{A}$ by $\mathfrak{s a}(\mathfrak{A})$ and the state space of $\mathfrak{A}$ by $\mathscr{S}(\mathfrak{A})$.

Notation 1.2. Let $\mathfrak{A}$ be a $C^{*}$-algebra. Let $L$ be a seminorm defined on a subspace of $\mathfrak{s a}(\mathfrak{A})$. Denote this subspace as $\operatorname{dom}(\mathrm{L})$ and set $\mathrm{L}(a)=\infty$ for all $a \in \mathfrak{s a}(\mathfrak{A}) \backslash \operatorname{dom}(\mathrm{L})$ so that $\operatorname{dom}(\mathrm{L})=\{a \in \mathfrak{s a}(\mathfrak{A}): \mathrm{L}(a)<\infty\}$.

If $\mathrm{L}$ is defined on a subspace of $\mathfrak{A}$, then we denote this subspace as $\operatorname{dom}(\mathrm{L})_{\mathfrak{A}}$ and set $\mathrm{L}(a)=\infty$ for all $a \in \mathfrak{A} \backslash \operatorname{dom}(\mathrm{L})_{\mathfrak{A}}$, so that $\operatorname{dom}(\mathrm{L})_{\mathfrak{A}}=\{a \in \mathfrak{A}: \mathrm{L}(a)<\infty\}$ and $\operatorname{dom}(\mathrm{L})=\operatorname{dom}(\mathrm{L})_{\mathfrak{A}} \cap \mathfrak{s a}(\mathfrak{A})$.

Definition $1.3(27,28,30])$. A compact quantum metric space $(\mathfrak{A}, \mathrm{L})$ is an ordered pair where $\mathfrak{A}$ is a unital $\mathrm{C}^{*}$-algebra with unit $1_{\mathfrak{A}}$ and $\mathrm{L}$ is a seminorm defined on a unital dense subspace of $\mathfrak{s a}(\mathfrak{A})$ such that:

(1) $\{a \in \mathfrak{s a}(\mathfrak{A}): \mathrm{L}(a)=0\}=\mathbb{R} 1_{\mathfrak{A}}$,

(2) the Monge-Kantorovich metric defined, for all two states $\varphi, \psi \in \mathscr{S}(\mathfrak{A})$, by

$$
\mathrm{mk}_{\mathrm{L}}(\varphi, \psi)=\sup \{|\varphi(a)-\psi(a)|: a \in \operatorname{dom}(\mathrm{L}), \mathrm{L}(a) \leqslant 1\}
$$

metrizes the weak* topology of $\mathscr{S}(\mathfrak{A})$,

(3) the seminorm $L$ is lower semi-continuous with respect to $\|\cdot\|_{\mathfrak{A}}$.

If $(\mathfrak{A}, \mathrm{L})$ is a compact quantum metric space, then we call the seminorm $\mathrm{L}$ a Lip-norm. 
REMARK 1.4. The density condition on $\operatorname{dom}(\mathrm{L})$ in the above definition condition guarantees that the map $\mathrm{mk}_{\mathrm{L}}$ is a metric (possibly taking value $+\infty$ ) on $\mathscr{S}(\mathfrak{A})$, which follows by continuity and linearity of states and the fact that every element of a $\mathrm{C}^{*}$-algebra is a linear combination of self-adjoint elements.

Also, we note that the above definition is more specialized than Rieffel's definition of a compact quantum metric space. Indeed, Rieffel's original definition allowed for order unit spaces and no lower semi-continuity was required. The purpose of the above definition is to place Rieffel's definition within the context of the order unit space of self-adjoint elements of a unital $\mathrm{C}^{*}$-algebra and to avoid any technical issues that arise from not requiring the Lip-norm to be lower semi-continuous, which are addressed in 28.

In Rieffel's pioneering work on compact quantum metric spaces [27], certain equivalent conditions were given for the requirement that the Monge-Kantorovich metric metrizes the weak* topology of the state space. These conditions provide a useful tool for verifying this difficult property. Further equivalences were given in [26]. The following theorem summarizes all known characterizations of Lip-norms and the proof uses both Arzelà-Ascoli theorem and the classical structure of $C(X)$ with $X$ compact metric and its associated Lipschitz seminorm, which, in part, explains the term "compact quantum metric space." Theorem $1.5(\sqrt{26}[28])$. Let $(\mathfrak{A}, \mathrm{L})$ be an ordered pair where $\mathfrak{A}$ is unital $C^{*}$-algebra and $\mathrm{L}$ is a lower semi-continuous seminorm defined on unital dense subspace of $\mathfrak{s a}(\mathfrak{A})$. The following are equivalent:

(1) (A, L) is a compact quantum metric space;

(2) the metric $\mathrm{mk}_{\mathrm{L}}$ is bounded and there exists $r \in \mathbb{R}, r>0$ such that the set

$$
\left\{a \in \operatorname{dom}(\mathrm{L}): \mathrm{L}(a) \leqslant 1 \text { and }\|a\|_{\mathfrak{A}} \leqslant r\right\}
$$

is compact in $\mathfrak{A}$ for $\|\cdot\|_{\mathfrak{A}}$;

(3) the set

$$
\left\{a+\mathbb{R} 1_{\mathfrak{A}} \in \mathfrak{s a}(\mathfrak{A}) / \mathbb{R} 1_{\mathfrak{A}}: a \in \operatorname{dom}(\mathrm{L}), \mathrm{L}(a) \leqslant 1\right\}
$$

is compact in $\mathfrak{s a}(\mathfrak{A}) / \mathbb{R} 1_{\mathfrak{A}}$ for $\|\cdot\|_{\mathfrak{s a}(\mathfrak{A}) / \mathbb{R} 1_{\mathfrak{A}}}$;

(4) there exists a state $\mu \in \mathscr{S}(\mathfrak{A})$ such that the set

$$
\{a \in \operatorname{dom}(\mathrm{L}): \mathrm{L}(a) \leqslant 1 \text { and } \mu(a)=0\}
$$

is compact in $\mathfrak{A}$ for $\|\cdot\|_{\mathfrak{A}}$;

(5) for all $\mu \in \mathscr{S}(\mathfrak{A})$ the set

$$
\{a \in \operatorname{dom}(\mathrm{L}): \mathrm{L}(a) \leqslant 1 \text { and } \mu(a)=0\}
$$

is compact in $\mathfrak{A}$ for $\|\cdot\|_{\mathfrak{A}}$.

Latrémolière's quantum Gromov-Hausdorff propinquity 18, 20, 21 is a distance on the class of compact quantum metric spaces and serves as noncommutative analogue to the Gromov-Hausdorff distance that has been proven to be an especially profitable contribution to noncommutative metric geometry by expanding the possibilities of continuous families of $\mathrm{C}^{*}$-algebras as well as extending the notion of finite-dimensional approximations $[17,32$. In order to obtain an analogue of the Gromov-Hausdorff distance on the class of compact quantum metric spaces, a form of the Leibniz inequality seems needed for 
the Lip-norm, as was first observed by Latrémolière in [20], which we provide a definition for now.

Definition $1.6([21])$. A $(C, D)$-quasi-Leibniz compact quantum metric space $(\mathfrak{A}, \mathrm{L})$, for some $C \geqslant 1$ and $D \geqslant 0$, is compact quantum metric space such that the seminorm $\mathrm{L}$ is a $(C, D)$-quasi-Leibniz Lip-norm, i.e. for all $a, b \in \operatorname{dom}(\mathrm{L})$ :

$$
\max \{\mathrm{L}(a \circ b), \mathrm{L}(\{a, b\})\} \leqslant C\left(\|a\|_{\mathfrak{A}} \mathrm{L}(b)+\|b\|_{\mathfrak{A}} \mathrm{L}(a)\right)+D \mathrm{~L}(a) \mathrm{L}(b),
$$

where $a \circ b=(a b+b a) / 2$ is the Jordan product and $\{a, b\}=(a b-b a) /(2 i)$ is the Lie product.

When $C=1, D=0$, we call $\mathrm{L}$ a Leibniz Lip-norm. When we do not specify $C$ and $D$, we call $(\mathfrak{A}, \mathrm{L})$ a quasi-Leibniz compact quantum metric space.

In particular, the quantum Gromov-Hausdorff propinquity produces a distance on the class of compact quantum metric spaces of Definition 1.6 with a desirable distance 0 property given, in part, by a *-isomorphism (see Theorem-Definition 1.7, (5)). The following serves as a summary of results we will use in this paper involving Latrémolière's propinquity.

THEOREM-DEFINITION 1.7 ([20|21] ). Let qLCQMS be the class of all quasi-Leibniz compact quantum metric spaces. There exists a class function $\wedge$ from qLCQMS $\times$ qLCQMS to $[0, \infty) \subseteq \mathbb{R}$ such that:

(1) for any $\left(\mathfrak{A}, \mathrm{L}_{\mathfrak{A}}\right),\left(\mathfrak{B}, \mathrm{L}_{\mathfrak{B}}\right) \in \mathrm{qLCQMS}$ we have

$$
\Lambda\left(\left(\mathfrak{A}, \mathrm{L}_{\mathfrak{A}}\right),\left(\mathfrak{B}, \mathrm{L}_{\mathfrak{B}}\right)\right) \leqslant \max \left\{\operatorname{diam}\left(\mathscr{S}(\mathfrak{A}), \mathrm{mk}_{\mathrm{L}_{\mathfrak{A}}}\right), \operatorname{diam}\left(\mathscr{S}(\mathfrak{B}), \mathrm{mk}_{\mathrm{L}_{\mathfrak{B}}}\right)\right\},
$$

(2) for any $\left(\mathfrak{A}, \mathrm{L}_{\mathfrak{A}}\right),\left(\mathfrak{B}, \mathrm{L}_{\mathfrak{B}}\right) \in \mathrm{qLCQMS}$ we have

$$
0 \leqslant \Lambda\left(\left(\mathfrak{A}, \mathrm{L}_{\mathfrak{A}}\right),\left(\mathfrak{B}, \mathrm{L}_{\mathfrak{B}}\right)\right)=\Lambda\left(\left(\mathfrak{B}, \mathrm{L}_{\mathfrak{B}}\right),\left(\mathfrak{A}, \mathrm{L}_{\mathfrak{A}}\right)\right),
$$

(3) for any $\left(\mathfrak{A}, \mathrm{L}_{\mathfrak{A}}\right),\left(\mathfrak{B}, \mathrm{L}_{\mathfrak{B}}\right),\left(\mathfrak{C}, \mathrm{L}_{\mathfrak{C}}\right) \in \mathrm{qLCQMS}$ we have

$$
\Lambda\left(\left(\mathfrak{A}, \mathrm{L}_{\mathfrak{A}}\right),\left(\mathfrak{C}, \mathrm{L}_{\mathfrak{C}}\right)\right) \leqslant \Lambda\left(\left(\mathfrak{A}, \mathrm{L}_{\mathfrak{A}}\right),\left(\mathfrak{B}, \mathrm{L}_{\mathfrak{B}}\right)\right)+\Lambda\left(\left(\mathfrak{B}, \mathrm{L}_{\mathfrak{B}}\right),\left(\mathfrak{C}, \mathrm{L}_{\mathfrak{C}}\right)\right),
$$

(4) for any $\left(\mathfrak{A}, \mathrm{L}_{\mathfrak{A}}\right),\left(\mathfrak{B}, \mathrm{L}_{\mathfrak{B}}\right) \in \mathrm{qLCQMS}$ and for any bridge $\gamma$ from $\mathfrak{A}$ to $\mathfrak{B}$ defined in [20, Definition 3.6], we have

$$
\Lambda\left(\left(\mathfrak{A}, \mathrm{L}_{\mathfrak{A}}\right),\left(\mathfrak{B}, \mathrm{L}_{\mathfrak{B}}\right)\right) \leqslant \lambda\left(\gamma \mid \mathrm{L}_{\mathfrak{A}}, \mathrm{L}_{\mathfrak{B}}\right),
$$

where $\lambda\left(\gamma \mid \mathrm{L}_{\mathfrak{A}}, \mathrm{L}_{\mathfrak{B}}\right)$ is defined in [20, Definition 3.17],

(5) for any $\left(\mathfrak{A}, \mathrm{L}_{\mathfrak{A}}\right),\left(\mathfrak{B}, \mathrm{L}_{\mathfrak{B}}\right) \in \mathrm{qLCQMS}$, we have:

$$
\Lambda\left(\left(\mathfrak{A}, \mathrm{L}_{\mathfrak{A}}\right),\left(\mathfrak{B}, \mathrm{L}_{\mathfrak{B}}\right)\right)=0
$$

if and only if $\left(\mathfrak{A}, \mathrm{L}_{\mathfrak{A}}\right)$ and $\left(\mathfrak{B}, \mathrm{L}_{\mathfrak{B}}\right)$ are quantum isometric, i.e. if and only if there exists a ${ }^{*}$-isomorphism $\pi: \mathfrak{A} \rightarrow \mathfrak{B}$ with $\mathrm{L}_{\mathfrak{B}} \circ \pi=\mathrm{L}_{\mathfrak{A}}$, and as quantum isometry is an equivalence relation, $\Lambda$ induces a metric on the class of equivalence classes up to quantum isometry of quasi-Leibniz compact quantum metric spaces,

(6) if $\Xi$ is a class function from $q \mathrm{qLQMS} \times \mathrm{qLCQMS}$ to $[0, \infty)$ which satisfies properties (2), (3) and (4) above, then

$$
\Xi\left(\left(\mathfrak{A}, \mathrm{L}_{\mathfrak{A}}\right),\left(\mathfrak{B}, \mathrm{L}_{\mathfrak{B}}\right)\right) \leqslant \Lambda\left(\left(\mathfrak{A}, \mathrm{L}_{\mathfrak{A}}\right),\left(\mathfrak{B}, \mathrm{L}_{\mathfrak{B}}\right)\right)
$$

for all $\left(\mathfrak{A}, \mathrm{L}_{\mathfrak{A}}\right)$ and $\left(\mathfrak{B}, \mathrm{L}_{\mathfrak{B}}\right)$ in $\mathrm{qLCQMS}$. 
Due to this Theorem-Definition, we may introduce the following convention.

Convention 1.8. Let CMS denote the class of all compact metric spaces. Let $\mathcal{A}$ be a subclass of CMS, then by $(\mathcal{A}, \mathrm{GH})$, we mean the class of all equivalence classes up to isometry of compact metric spaces topologized by the quotient topology induced by the Gromov-Hausdorff distance, GH [7, Section 7.3], for which GH induces a metric on this quotient space. And, when we let $\left(X, \mathrm{~d}_{X}\right) \in(\mathcal{A}, \mathrm{GH})$, we implicitly mean the equivalence class of $\left(X, \mathrm{~d}_{X}\right)$ with respect to isometry.

By (qLCQMS, $\Lambda$ ), we mean the class of all equivalence classes up to quantum isometry of Theorem-Definition 1.7 topologized by the quotient topology induced by the quantum Gromov-Hausdorff propinquity, $\Lambda$. And, when we take $(\mathfrak{A}, \mathrm{L}) \in(\mathrm{qLCQMS}, \Lambda)$, we implicitly mean the equivalence class of $(\mathfrak{A}, \mathrm{L})$ with respect to quantum isometry.

We also have the following theorem that establishes the quantum Gromov-Hausdorff propinquity as a noncommutative analogue of the Gromov-Hausdorff distance on compact metric spaces.

Theorem 1.9 ([20, Theorem 6.6, Corollary 6.4] and [29, Theorem 13.6]). If given compact metric spaces $\left(X, \mathrm{~d}_{X}\right),\left(Y, \mathrm{~d}_{Y}\right)$ and $\mathrm{L}_{\mathrm{d}_{X}}, \mathrm{~L}_{\mathrm{d}_{Y}}$ denote their respective Lipschitz seminorms, then

$$
\Lambda\left(\left(C(X), \mathrm{L}_{\mathrm{d}_{X}}\right),\left(C(Y), \mathrm{L}_{\mathrm{d}_{Y}}\right)\right) \leqslant \mathrm{GH}\left(\left(X, \mathrm{~d}_{X}\right),\left(Y, \mathrm{~d}_{Y}\right)\right),
$$

where GH is the Gromov-Hausdorff distance [7, Section 7.3].

Moreover, with Convention 1.8, the class map

$$
\Gamma:\left(X, \mathrm{~d}_{X}\right) \in(\mathrm{CMS}, \mathrm{GH}) \mapsto\left(C(X), \mathrm{L}_{\mathrm{d}_{X}}\right) \in(\mathrm{qLCQMS}, \Lambda)
$$

is a homeomorphism onto its image.

A main goal of this paper is to generalize the continuity of $\Gamma$ in this theorem to matrix-valued continuous functions.

2. Quantum metrics on standard homogeneous $\mathbf{C}^{*}$-algebras. Given a compact metric space $\left(X, \mathrm{~d}_{X}\right)$ and a finite-dimensional $\mathrm{C}^{*}$-algebra $\mathfrak{A}$, the task of equipping $C(X, \mathfrak{A})$ with a Lip-norm may at first seem obvious since we could define the quantity

$$
L(a)=\sup _{x, y \in X, x \neq y} \frac{\|a(x)-a(y)\|_{\mathfrak{A}}}{\mathrm{d}_{X}(x, y)} \text { for all } a \in C(X, \mathfrak{A}) .
$$

However, an immediate issue with this quantity is that the kernel of $L$ is not $\mathbb{C} 1_{C(X, \mathfrak{A})}$ if $\operatorname{dim}(\mathfrak{A})>1$. Thus, we would immediately not satisfy the definition of the Lip-norm and Theorem 1.5 (the theorem that, in part, motivates the term "compact quantum metric space") would be unavailable to us. Hence, in this section, we present various remedies to this deficit by coupling the quantity $L$ with other quantities and choosing different norms on $\mathfrak{A}$ for the quantity in the numerator of $L$ to make a Lip-norm. We will discuss the advantages of each construction and, in the process, provide new Lip-norms on $C(X)$ itself.

First, we explicitly define what we mean by a standard homogeneous $\mathrm{C}^{*}$-algebra with a remark afterward explaining this definition. 
Definition 2.1. A unital separable $\mathrm{C}^{*}$-algebra $\mathfrak{B}$ is a standard homogeneous $\mathrm{C}^{*}$-algebra if there exists a compact metric space $\left(X, \mathrm{~d}_{X}\right)$ and a finite-dimensional $\mathrm{C}^{*}$-algebra $\mathfrak{A}$ such that $\mathfrak{B}=C(X, \mathfrak{A})$, which is the $\mathrm{C}^{*}$-algebra of continuous $\mathfrak{A}$-valued functions on $X$ with point-wise algebraic and adjoint operations induced by $\mathfrak{A}$, supremum norm, and the unit is the constant $1_{\mathfrak{A}}$ function on $X$.

REMARK 2.2. The reason we assume $X$ is compact metric in the previous definition is because the $\mathrm{C}^{*}$-algebra of a compact quantum metric space is always unital by definition and separable by [18, Proposition 2.11]. Indeed, if $X$ is compact Hausdorff and $\mathfrak{A}$ is finite-dimensional and $C(X, \mathfrak{A})$ is separable, then its state space is compact by unital and metrizable as the unit ball of a the dual of a separable Banach space is metrizable in the weak* topology. However, $X$ embeds homeomorphically into the state space of $C(X, \mathfrak{A})$ (see proof of Proposition 3.6), which induces a metric on $X$ that agrees with its topology. The terminology "standard" is taken from [4, Section IV.1.4].

Now, we define some of the seminorms we consider throughout this paper. We note that we define them in the more general setting than Definition 2.1, in which the $\mathrm{C}^{*}$ algebra $\mathfrak{A}$ in $C(X, \mathfrak{A})$ need not be finite-dimensional. And, in fact, we can and do prove many interesting properties about the seminorms of the following definition without the assumption that $\mathfrak{A}$ is finite-dimensional. We only assume $\mathfrak{A}$ is finite-dimensional when it is necessary in Theorem 2.10 in that this theorem, in part, provides an equivalence for finite-dimensionality of $\mathfrak{A}$. This thus shows that our seminorms are natural choices for Lip-norms on $C(X, \mathfrak{A})$ in the case when $\mathfrak{A}$ is finite-dimensional.

Definition 2.3. Let $\left(X, \mathrm{~d}_{X}\right)$ be a compact metric space and let $\mathfrak{A}$ be a unital $\mathrm{C}^{*}$-algebra. Let $C(X, \mathfrak{A})$ denote the unital $\mathrm{C}^{*}$-algebra of continuous $\mathfrak{A}$-valued functions on $X$ with point-wise algebraic and adjoint operations induced by $\mathfrak{A}$, supremum norm over $X$ and with $1_{C(X, \mathfrak{A})}$ the constant $1_{\mathfrak{A}}$ function over $X$. Note that $\mathfrak{s a}(C(X, \mathfrak{A}))=C(X, \mathfrak{s a}(\mathfrak{A}))$.

Let $C\left(X, \mathbb{C}_{\mathfrak{A}}\right)=\left\{a \in C(X, \mathfrak{A}): a(x) \in \mathbb{C}_{\mathfrak{A}}\right.$ for all $\left.x \in X\right\}$. Define

$$
l_{\mathrm{d}_{X}}^{(\mathrm{n})}(a)=\sup \left\{\frac{\|a(x)-a(y)\|_{\mathrm{n}}}{\mathrm{d}_{X}(x, y)}: x, y \in X, x \neq y\right\} \text { for all } a \in \mathfrak{s a}(C(X, \mathfrak{A}))
$$

where $\|\cdot\|_{\text {n }}$ denotes any norm on $\mathfrak{A}$.

Let $\mathrm{L}_{\mathrm{d}_{X}}^{(\mathrm{n}), q}$ denote the following:

(1) if $q=C(X)$, then for all $a \in C(X, \mathfrak{A})$ let

$$
\mathrm{L}_{\mathrm{d}_{X}}^{(\mathrm{n}), q}(a)=\max \left\{l_{\mathrm{d}_{X}}^{(\mathrm{n})}(a),\left\|a+C\left(X, \mathbb{C} 1_{\mathfrak{A}}\right)\right\|_{C(X, \mathfrak{A}) / C\left(X, \mathbb{C} 1_{\mathfrak{A}}\right)}\right\} ;
$$

(2) if $q=\mathbb{C}$, then for all $a \in C(X, \mathfrak{A})$ let

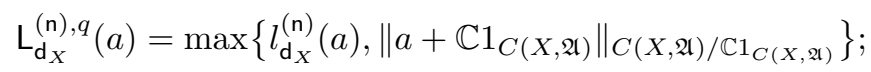

(3) if $\mu \in \mathscr{S}(C(X, \mathfrak{A}))$ is any state and $q=\mu$, then for all $a \in C(X, \mathfrak{A})$ let

$$
\mathrm{L}_{\mathrm{d}_{X}}^{(\mathrm{n}), q}(a)=\max \left\{l_{\mathrm{d}_{X}}^{(\mathrm{n})}(a),\left\|a-\mu(a) 1_{C(X, \mathfrak{A})}\right\|_{C(X, \mathfrak{A})}\right\} .
$$

If $\mathfrak{A}=\mathbb{C}, \mathrm{n}=\mathbb{C}$ is the usual norm on $\mathbb{C}$, and $q=C(X)$, then we note that $\mathrm{L}_{\mathrm{d}_{X}, q}^{(\mathrm{n})}=l_{\mathrm{d}_{X}}^{(\mathrm{n})}$ and denote this by $L_{d_{X}}$. 
As a convention, when not specified, $\mathrm{n}$ and $q$ will simply be allowed to be any of the above cases.

We note that the constructions of the above definition are related to the construction of a norm in [16. Proposition 4.4], where the non-unital case is considered, in which $X$ is a locally compact separable metric space. However, the seminorm used there is a norm and uses the norm on $C_{0}(X, \mathfrak{A})$ in place of $q$ above. Hence, the norm of [16, Proposition 4.4] applied in our setting would vanish only at 0 , which would not provide a possibility for a Lip-norm. Thus, the fact that we are left to rely on the above choices of $q$ does require us to do more work to prove that the seminorms of Definition 2.3 form Lip-norms if and only if $\mathfrak{A}$ is finite-dimensional.

When $\mathfrak{A}$ is a finite-dimensional $\mathrm{C}^{*}$-algebra, there are many standard norms that can be placed on $\mathfrak{A}$, which are automatically equivalent by finite-dimensionality. Later in Sections 3 and 4 we will utilize other norms aside from the $\mathrm{C}^{*}$-norm. But, for now, let us focus on the algebraic properties of the domain of the seminorms of Definition 2.3 Note that Proposition 2.4 does not assume finite-dimensionality of $\mathfrak{A}$ in $C(X, \mathfrak{A})$ and thus finite-dimensionality assumption on $\mathfrak{A}$ does not appear until Theorem 2.10, where it is, in fact, a necessity.

Proposition 2.4. Let $\left(X, \mathrm{~d}_{X}\right)$ be a compact metric space and let $\mathfrak{A}$ be a unital $C^{*}$-algebra and let $\mu \in \mathscr{S}(C(X, \mathfrak{A}))$ be a state.

With notation from Definition 2.3. if $\|\cdot\|_{\mathrm{n}}$ is a norm on $\mathfrak{A}$ that is equivalent to the $C^{*}$-norm $\|\cdot\|_{\mathfrak{A}}$, then $\operatorname{ker} \mathrm{L}_{\mathrm{d}_{X}}^{(\mathrm{n}), q}=\mathbb{C} 1_{C(X, \mathfrak{A})}$ and $\operatorname{dom}\left(\mathrm{L}_{\mathrm{d}_{X}}^{(\mathrm{n}), q}\right)_{C(X, \mathfrak{A})}$ is a unital ${ }^{*}$-subalgebra of $C(X, \mathfrak{A})$.

Furthermore, if $M>0, N>0$ such that $M\|\cdot\|_{\mathrm{n}} \leqslant\|\cdot\|_{\mathfrak{A}} \leqslant N\|\cdot\|_{\mathrm{n}}$, then:

(1) if $q$ is either $C(X)$ or $\mathbb{C}$, then $\mathrm{L}_{\mathrm{d}_{X}}^{(\mathrm{n}), q}$ is a $(N / M, 0)$-quasi-Leibniz seminorm;

(2) if $q=\mu$, then $\mathrm{L}_{\mathrm{d}_{X}}^{(\mathrm{n}), q}$ is a $(\max \{N / M, 2\}, 0)$-quasi-Leibniz seminorm.

Proof. For ker $\mathrm{L}_{\mathrm{d}_{X}, q}^{(\mathrm{n}), q}=\mathbb{C} 1_{C(X, \mathfrak{A})}$, first note that if $a \in \mathbb{C} 1_{C(X, \mathfrak{A})} \subseteq C\left(X, \mathbb{C} 1_{\mathfrak{A}}\right)$, then since $a$ is constant, $l_{\mathrm{d}_{X}}^{(\mathrm{n})}(a)=0$ and, for any choice of $q$, the second expression in the definition of $\mathrm{L}_{\mathrm{d}_{X}}^{(\mathrm{n}), q}$ is also 0 and so $\mathrm{L}_{\mathrm{d}_{X}}^{(\mathrm{n}), q}(a)=0$. Hence, we have $\operatorname{ker} \mathrm{L}_{\mathrm{d}_{X}}^{(\mathrm{n}), q} \supseteq \mathbb{C}_{C(X, \mathfrak{A})}$.

Next, let $a \in \operatorname{ker}_{\mathrm{d}_{X}}^{(\mathrm{n}), q}$. First, consider the case when $q=\mathbb{C}$ or $q=\mu$. Since $\mathrm{L}_{\mathrm{d}_{X}}^{(\mathrm{n}), q}(a)=0$, then

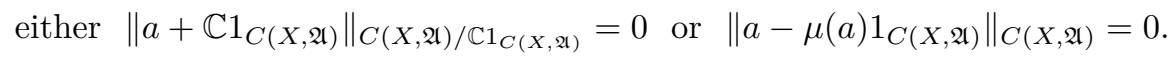

In either case, we have $a \in \mathbb{C} 1_{C(X, \mathfrak{A})}$ and so $\operatorname{ker~}_{\mathrm{d}_{X}}^{(\mathrm{n}), q}=\mathbb{C} 1_{C(X, \mathfrak{A})}$. Second, assume that $q=C(X)$. If $\mathrm{L}_{\mathrm{d}_{X}}^{(\mathrm{n}), q}(a)=0$, then $l_{\mathrm{d}_{X}}^{(\mathrm{n})}(a)=0$ implies that $a$ is constant. However, the expression $\left\|a+C\left(X, \mathbb{C} 1_{\mathfrak{A}}\right)\right\|_{C(X, \mathfrak{A}) / C\left(X, \mathbb{C} 1_{\mathfrak{A}}\right)}=0$ implies that $a(x) \in \mathbb{C} 1_{\mathfrak{A}}$ for all $x \in X$. Thus $a$ is a constant scalar and so $a \in \mathbb{C} 1_{C(X, \mathfrak{A})}$, which implies that $\operatorname{ker}_{\mathrm{d}_{X}}^{(\mathrm{n}), q}=\mathbb{C} 1_{C(X, \mathfrak{A})}$.

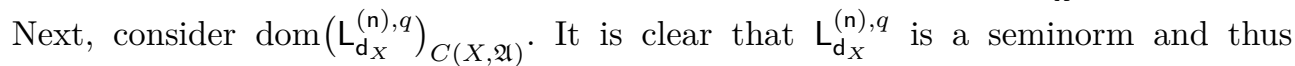
$\operatorname{dom}\left(\mathrm{L}_{\mathrm{d}_{X}}^{(\mathrm{n}), q}\right)_{C(X, \mathfrak{A})}$ is a subspace of $C(X, \mathfrak{A})$, and we have already shown that it is unital. 
For subalgebra, since $\|\cdot\|_{\mathrm{n}}$ is equivalent to $\|\cdot\|_{\mathfrak{A}}$, there exist $M, N>0$ such that $M\|\cdot\|_{\mathrm{n}} \leqslant\|\cdot\|_{\mathfrak{A}} \leqslant N\|\cdot\|_{\mathrm{n}}$. Let $a, b \in \operatorname{dom}\left(\mathrm{L}_{\mathrm{d}_{X}}^{(\mathrm{n}), q}\right)_{C(X, \mathfrak{A})}$. We then have:

$$
\begin{aligned}
\|a b(x)-a b(y)\|_{\mathrm{n}} & \leqslant \frac{1}{M}\left(\|a b(x)-a(x) b(y)\|_{\mathfrak{A}}+\|a(x) b(y)-a(y) b(y)\|_{\mathfrak{A}}\right) \\
& \leqslant \frac{1}{M}\left(\|a(x)\|_{\mathfrak{A}} \cdot\|b(x)-b(y)\|_{\mathfrak{A}}+\|a(x)-a(y)\|_{\mathfrak{A}} \cdot\|b(y)\|_{\mathfrak{A}}\right) \\
& \leqslant \frac{1}{M}\left(\|a\|_{C(X, \mathfrak{A})} \cdot\|b(x)-b(y)\|_{\mathfrak{A}}+\|a(x)-a(y)\|_{\mathfrak{A}} \cdot\|b\|_{C(X, \mathfrak{A})}\right) \\
& =\frac{N}{M}\left(\|a\|_{C(X, \mathfrak{A})} \cdot\|b(x)-b(y)\|_{\mathrm{n}}+\|a(x)-a(y)\|_{\mathrm{n}} \cdot\|b\|_{C(X, \mathfrak{A})}\right)
\end{aligned}
$$

for all $x, y \in X$. Thus, since $l_{\mathrm{d}_{X}}^{(\mathrm{n})}(a), l_{\mathrm{d}_{X}}^{(\mathrm{n})}(b)<\infty$, we have $l_{\mathrm{d}_{X}}^{(\mathrm{n})}(a b)<\infty$. Furthermore, the seminorm $l_{\mathrm{d}_{X}}^{(\mathrm{n})}$ is $(N / M, 0)$-quasi-Leibniz. And, clearly, the expression determined by $q$ is finite on all of $C(X, \mathfrak{A})$. Hence, the unital subspace $\operatorname{dom}\left(\mathrm{L}_{\mathrm{d}_{X}}^{(\mathrm{n}), q}\right)_{C(X, \mathfrak{A})}$ is a subalgebra.

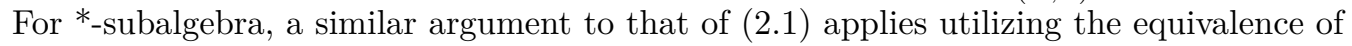
norms and the fact that the adjoint is an isometry with respect to the $\mathrm{C}^{*}$-norm.

Finally, note that both quotient norms $\|\cdot\|_{C(X, \mathfrak{A}) / \mathbb{C} 1_{C(X, \mathfrak{A})}}$ and $\|\cdot\|_{C(X, \mathfrak{A}) / C\left(X, \mathbb{C} 1_{\mathfrak{A}}\right)}$ are Leibniz by $\left[31\right.$, Theorem 3.1] since both $\mathbb{C} 1_{C(X, \mathfrak{A})}$ and $C\left(X, \mathbb{C} 1_{\mathfrak{A}}\right)$ are unital $\mathrm{C}^{*}$ subalgebras of $C(X, \mathfrak{A})$. Also, note that for equivalent norms $M \leqslant N$ or $(N / M) \geqslant 1$ lest we reach a contradiction. This along with expression (2.1) provides statement (1) of this proposition.

For statement (2), we note that the function $a \in C(X, \mathfrak{A}) \longmapsto \mu(a) 1_{C(X, \mathfrak{A})}$ is a conditional expectation onto $\mathbb{C} 1_{C(X, \mathfrak{A})}$. Hence, by $[3$, Lemma 3.2], we conclude that the seminorm

$$
a \in C(X, \mathfrak{A}) \longmapsto\left\|a-\mu(a) 1_{C(X, \mathfrak{A})}\right\|_{C(X, \mathfrak{A})}
$$

is $(2,0)$-quasi-Leibniz, and this establishes statement (2) with expression (2.1).

We now move onto the analytic properties of the seminorm $\mathrm{L}_{\mathrm{d}_{X}}^{(\mathrm{n}), q}$ on $C(X, \mathfrak{A})$ such as lower semi-continuity and density of the domain. Towards this goal, in Lemma 2.5, we prove these properties in the classical case $C(X)$ equipped with its standard Lipschitz seminorm $L_{d_{X}}$, which is a well-known result but useful for the proof of the lemma that follows.

Lemma 2.5. Consider the $C^{*}$-algebra $C(X)$. The Lipschitz seminorm $\mathrm{L}_{\mathrm{d}_{X}}$ is lower semicontinuous on $C(X)$ with respect to $\|\cdot\|_{C(X)}$ and its domain $\operatorname{dom}\left(\mathrm{L}_{\mathrm{d}_{X}}\right)_{C(X)}$ is dense.

Proof. First, we check lower semi-continuity of $\mathrm{L}_{\mathrm{d}_{X}}$. Fix $x, y \in X$. Note that the map $\mathrm{L}_{x, y}: f \in C(X) \longmapsto \frac{|f(x)-f(y)|}{\mathrm{d}_{X}(x, y)} \in \mathbb{R}$ is continuous. But, we have $\mathrm{L}_{\mathrm{d}_{X}}(f)=\sup \left\{\mathrm{L}_{x, y}(f)\right.$ : $x, y \in X\}$. Hence, since a supremum of real-valued lower semi-continuous functions is lower semi-continuous, we see that $\mathrm{L}_{\mathrm{d}_{X}}$ is lower semi-continuous.

Next, we prove density of $\operatorname{dom}\left(\mathrm{L}_{\mathrm{d}_{X}}\right)_{C(X)}$ in $C(X)$. By Proposition 2.4, $\operatorname{dom}\left(\mathrm{L}_{\mathrm{d}_{X}}\right)_{C(X)}$ is a unital *-subalgebra of $C(X)$. Now, fix $a, b \in X, a \neq b$ and consider the function on $X$ defined by $a_{\mathrm{d}}(x)=\mathrm{d}_{X}(a, x)$ for all $x \in X$. Clearly, the function $a_{\mathrm{d}} \in C(X)$. Also, we see for $x, y \in X$ that $\left|a_{\mathrm{d}}(x)-a_{\mathrm{d}}(y)\right|=\left|\mathrm{d}_{X}(a, x)-\mathrm{d}_{X}(a, y)\right| \leqslant \mathrm{d}_{X}(x, y)$. Hence, the function $a_{\mathrm{d}} \in \operatorname{dom}\left(\mathrm{L}_{\mathrm{d}_{X}}\right)_{C(X)}$. Finally, $a_{\mathrm{d}}(b)>0=a_{\mathrm{d}}(a)$, which implies that $\operatorname{dom}\left(\mathrm{L}_{\mathrm{d}_{X}}\right)_{C(X)}$ 
separates the points of $X$. Therefore, the proof is complete by 35 , Stone-Weierstrass Theorem 44.5].

Now, we are prepared to generalize the results of Lemma 2.5 when $\mathbb{C}$ is replaced by any unital $\mathrm{C}^{*}$-algebra. We utilize the argument outlined in $[15$, Theorem 3.4] to obtain the following.

Lemma 2.6. Let $\left(X, \mathrm{~d}_{X}\right)$ be a compact metric space and let $\mathfrak{A}$ be a unital $C^{*}$-algebra. Using notation from Definition 2.3. if $\|\cdot\|_{\mathrm{n}}$ is a norm on $\mathfrak{A}$ that is equivalent to the $C^{*}$ norm $\|\cdot\|_{\mathfrak{A}}$, then the seminorm $\mathrm{L}_{\mathrm{d}_{X}}^{(\mathrm{n}), q}$ is lower semi-continuous with respect to $\|\cdot\|_{C(X, \mathfrak{A})}$ and the sets $\operatorname{dom}\left(\mathrm{L}_{\mathrm{d}_{X}}^{(\mathrm{n}), q}\right)_{C(X, \mathfrak{A})}$ and $\operatorname{dom}\left(\mathrm{L}_{\mathrm{d}_{X}}^{(\mathrm{n}), q}\right)$ are dense in $C(X, \mathfrak{A})$ and $\mathfrak{s a}(C(X, \mathfrak{A}))$, respectively.

Proof. Semi-continuity follows as in the proof of Lemma 2.5 along with the fact that $\|\cdot\|_{\mathrm{n}}$ is equivalent to $\|\cdot\|_{\mathfrak{A}}$.

For density of $\operatorname{dom}\left(\mathrm{L}_{\mathrm{d}_{X}}^{(\mathrm{n}), q}\right)_{C(X, \mathfrak{A})}$ in $C(X, \mathfrak{A})$, let $f \in C(X, \mathfrak{A})$. Let $\varepsilon>0$. As $X$ is compact, $f$ is uniformly continuous, and thus there exists $\delta>0$ such that:

$$
\mathrm{d}_{X}(x, y)<\delta \quad \Longrightarrow \quad\|f(x)-f(y)\|_{\mathfrak{A}}<\varepsilon / 2
$$

for all $x, y \in X$. Define $U(y, \delta / 2)=\left\{x \in X: \mathrm{d}_{X}(x, y)<\delta / 2\right\}$ for all $y \in X$. Again, as $X$ is compact, the open cover $\{U(y, \delta / 2) \subseteq X: y \in X\}$ of $X$ has a finite subcover of $X$ given by $y_{1}, \ldots, y_{n} \in X$ such that $\bigcup_{k=1}^{n} U\left(y_{k}, \delta / 2\right)=X$. Since $X$ is compact Hausdorff, there exists a partition of unity with respect to the cover $\left\{U\left(y_{1}, \delta / 2\right), \ldots, U\left(y_{n}, \delta / 2\right)\right\}$ by 5. Proposition IX.4.3.3]. In particular, for each $k \in\{1, \ldots, n\}$, there exists a continuous function $p_{k}: X \rightarrow[0,1]$ such that $\left\{x \in X: p_{k}(x)>0\right\} \neq \emptyset$ and if we define $V_{k}=\{x \in X$ : $\left.p_{k}(x)>0\right\}$, then $\left\{V_{1}, \ldots, V_{n}\right\}$ is an open cover of $X$ and $V_{k} \subseteq{\overline{V_{k}}}^{\mathrm{d}_{X}} \subseteq U\left(y_{k}, \delta / 2\right)$ for each $k \in\{1, \ldots, n\}$. Furthermore, we have $\sum_{k=1}^{n} p_{k}=1_{C(X)}$, which is the constant 1 function on $X$.

Now, note that by definition of $l_{\mathrm{d}_{X}}^{(\mathrm{n})}$, we see that if $g \in C(X)$ and $\mathrm{L}_{\mathrm{d}_{X}}(g)<\infty$, then for any $a \in \mathfrak{A}, a \cdot g \in C(X, \mathfrak{A})$, where $a \cdot g: x \in X \mapsto a \cdot g(x) \in \mathfrak{A}$, and $l_{\mathrm{d}_{X}}^{(\mathrm{n})}(a \cdot g)=\|a\|_{\mathrm{n}} \cdot \mathrm{L}_{\mathrm{d}_{X}}(g)<\infty$. Next, for all $k \in\{1, \ldots, n\}$, fix some $x_{k} \in V_{k}$. Let $k \in$ $\{1, \ldots, n\}$. If $f\left(x_{k}\right)=0$, then clearly $f\left(x_{k}\right) p_{k} \in \operatorname{dom}\left(\mathrm{L}_{\mathrm{d}_{X}}^{(\mathrm{n}), q}\right)_{C(X, \mathfrak{A})}$. If $f\left(x_{k}\right) \neq 0$, then let $q_{k} \in \operatorname{dom}\left(\mathrm{L}_{\mathrm{d}_{X}}\right)_{C(X)}$ such that $\left\|p_{k}-q_{k}\right\|_{C(X)}<\varepsilon /\left(2 n \cdot\left\|f\left(x_{k}\right)\right\|_{\mathfrak{A}}\right)$ by Lemma 2.5 Furthermore, by the comments at the beginning of this paragraph, $f\left(x_{k}\right) \cdot q_{k} \in \operatorname{dom}\left(\mathrm{L}_{\mathrm{d}_{X}}^{(\mathrm{n}), q}\right)_{C(X, \mathfrak{A})}$ and

$$
\left\|f\left(x_{k}\right) \cdot p_{k}-f\left(x_{k}\right) \cdot q_{k}\right\|_{C(X, \mathfrak{A})}=\left\|f\left(x_{k}\right)\right\|_{\mathfrak{A}} \cdot\left\|p_{k}-q_{k}\right\|_{C(X)}<\varepsilon /(2 n) .
$$

Thus

$$
\left\|\sum_{k=1}^{n} f\left(x_{k}\right) \cdot p_{k}-\sum_{k=1}^{n} f\left(x_{k}\right) \cdot q_{k}\right\|_{C(X, \mathfrak{A})}<n \cdot \varepsilon /(2 n)=\varepsilon / 2 .
$$

Next, define $f_{p}=\sum_{k=1}^{n} f\left(x_{k}\right) \cdot p_{k} \in C(X, \mathfrak{A})$ and $f_{\varepsilon}=\sum_{k=1}^{n} f\left(x_{k}\right) \cdot q_{k}$. Note that $f_{\varepsilon} \in \operatorname{dom}\left(\mathrm{L}_{\mathrm{d}_{X}}^{(\mathrm{n}), q}\right)_{C(X, \mathfrak{A})}$ since $\mathrm{L}_{\mathrm{d}_{X}}^{(\mathrm{n}), q}$ is a seminorm, and note that $\left\|f_{p}-f_{\varepsilon}\right\|_{C(X, \mathfrak{A})}<\varepsilon / 2$.

Now, let $x \in X$. Since $\left\{V_{1}, \ldots, V_{n}\right\}$ is a cover of $X$, we see that $\emptyset \neq\{l \in\{1, \ldots, n\}$ : $\left.x \in V_{l}\right\}=\left\{l \in\{1, \ldots, n\}: p_{l}(x)>0\right\}$. Let $\left\{l_{1}, \ldots, l_{m}\right\}=\left\{l \in\{1, \ldots, n\}: p_{l}(x)>0\right\}$, 
and in particular, we have $p_{l}(x)=0$ if $l \in\{1, \ldots, n\} \backslash\left\{l_{1}, \ldots, l_{m}\right\}$. Since $x \in V_{l_{j}} \subseteq$ $U\left(y_{l_{j}}, \delta / 2\right)$, for all $j \in\{1, \ldots, m\}$, we have $\mathrm{d}_{X}\left(x, x_{l_{j}}\right)<\delta$ for all $j \in\{1, \ldots, m\}$. Hence by expression $(2.2)$, we gather:

$$
\begin{aligned}
& \left\|f(x)-f_{p}(x)\right\|_{\mathfrak{A}}=\left\|\left(\sum_{k=1}^{n} p_{k}(x)\right) \cdot f(x)-\sum_{k=1}^{n} f\left(x_{k}\right) \cdot p_{k}(x)\right\|_{\mathfrak{A}} \\
& =\left\|\left(\sum_{k=1}^{n} p_{k}(x) \cdot f(x)\right)-\sum_{k=1}^{n} f\left(x_{k}\right) \cdot p_{k}(x)\right\|_{\mathfrak{A}} \leqslant \sum_{k=1}^{n} p_{k}(x) \cdot\left\|f(x)-f\left(x_{k}\right)\right\|_{\mathfrak{A}} \\
& =\sum_{j=1}^{m} p_{l_{j}}(x) \cdot\left\|f(x)-f\left(x_{l_{j}}\right)\right\|_{\mathfrak{A}}<\sum_{j=1}^{m} p_{l_{j}}(x) \cdot \varepsilon / 2 \leqslant 1 \cdot \varepsilon / 2
\end{aligned}
$$

since $\sum_{k=1}^{n} p_{k}=1_{C(X)}$. As $x \in X$ was arbitrary, we have $\left\|f-f_{p}\right\|_{C(X, \mathfrak{A})} \leqslant \varepsilon / 2$, which implies that $\left\|f-f_{\varepsilon}\right\|_{C(X, \mathfrak{A})} \leqslant\left\|f-f_{p}\right\|_{C(X, \mathfrak{A})}+\left\|f_{p}-f_{\varepsilon}\right\|_{C(X, \mathfrak{A})}<\varepsilon / 2+\varepsilon / 2=\varepsilon$, where $f_{\varepsilon} \in \operatorname{dom}\left(\mathrm{L}_{\mathrm{d}_{X}}^{(\mathrm{n}), q}\right)_{C(X, \mathfrak{A})}$, which establishes that $\operatorname{dom}\left(\mathrm{L}_{\mathrm{d}_{X}}^{(\mathrm{n}), q}\right)_{C(X, \mathfrak{A})}$ is dense in $C(X, \mathfrak{A})$.

Finally, $\operatorname{dom}\left(\mathrm{L}_{\mathrm{d}_{X}}^{(\mathrm{n}), q}\right)_{C(X, \mathfrak{A})}$ is a *-subalgebra of $C(X, \mathfrak{A})$ by Proposition 2.4 hence $\operatorname{dom}\left(\mathrm{L}_{\mathrm{d}_{X}}^{(\mathrm{n}), q}\right)_{C(X, \mathfrak{A})} \cap \mathfrak{s a}(C(X, \mathfrak{A}))=\operatorname{dom}\left(\mathrm{L}_{\mathrm{d}_{X}}^{(\mathrm{n}), q}\right)$ is dense in $\mathfrak{s a}(C(X, \mathfrak{A}))$. Indeed, let $a \in$ $\mathfrak{s a}(C(X, \mathfrak{A}))$. There exists a sequence $\left(a_{n}\right)_{n \in \mathbb{N}} \subset \operatorname{dom}\left(\mathrm{L}_{\mathrm{d}_{X}}^{(\mathrm{n}), q}\right)_{C(X, \mathfrak{A})}$ that converges to $a$. However, as $\operatorname{dom}\left(\mathrm{L}_{\mathrm{d}_{X}}^{(\mathrm{n}), q}\right)_{C(X, \mathfrak{A})}$ is a *-subalgebra, we have

$$
\left(\frac{a_{n}+a_{n}^{*}}{2}\right)_{n \in \mathbb{N}} \subset \operatorname{dom}\left(\mathrm{L}_{\mathrm{d}_{X}}^{(\mathrm{n}), q}\right)_{C(X, \mathfrak{A})} \cap \mathfrak{s a}(C(X, \mathfrak{A}))=\operatorname{dom}\left(\mathrm{L}_{\mathrm{d}_{X}}^{(\mathrm{n}), q}\right)
$$

and converges to $\frac{a+a^{*}}{2}=\frac{a+a}{2}=a$, which completes the proof.

REMARK 2.7. In Lemma 2.6 the proof of the density of $\operatorname{dom}\left(\mathrm{L}_{\mathrm{d}_{X}}^{(\mathrm{n}), q}\right)_{C(X, \mathfrak{A})}$ did not utilize the $\mathrm{C}^{*}$-algebra structure of $\mathfrak{A}$, and this density result would be true if $\mathfrak{A}$ was any normed space.

We are on our way to the final steps in proving that we have compact quantum metric spaces. There are several ways to approach this. We will use [27, Theorem 1.9] (this is equivalence (2) of Theorem 1.5), which is Rieffel's first characterization of compact quantum metric spaces and characterizes when the weak* topology on the state space is metrized by the Monge-Kantorovich metric (the quantum metric) of Definition 1.3 by a fascinating application of the Arzelà-Ascoli Theorem. The reason we use equivalence (2) of Theorem 1.5 is because it requires us to study the diameter of the Monge-Kantorovich metric, which will provide greater insight into the results of Section 3 (see Remark 3.8). This is Proposition 2.9 However, we first prove a lemma which is a likely well-known fact about a characterization of pure states on $C(X, \mathfrak{A})$.

LEMmA 2.8. Let $\left(X, \mathrm{~d}_{X}\right)$ be a compact metric space and $\mathfrak{A}$ be a unital $C^{*}$-algebra. Let $x \in X$ and $\phi$ be a pure state on $\mathfrak{A}$. If we define $\phi_{x}(a)=\phi(a(x))$ for all $a \in C(X, \mathfrak{A})$, then $\phi_{x}$ is a pure state on $C(X, \mathfrak{A})$.

Furthermore, if $\mu$ is a pure state on $C(X, \mathfrak{A})$, then there exists $x \in X$ and a pure state $\phi$ on $\mathfrak{A}$ such that $\mu=\phi_{x}$. 
Proof. It is clear that $\phi_{x}$ defines a state on $C(X, \mathfrak{A})$ for all $x \in X$ and all pure states $\phi$ on $\mathfrak{A}$. We show that $\phi_{x}$ is a pure state by verifying the final statement in the lemma.

Let $\mathfrak{A} \odot C(X)$ denote the algebra over $\mathbb{C}$ formed over the algebraic tensor product of $\mathfrak{A}$ and $C(X)\left[6\right.$, Section 3.1]. Let $\mathfrak{A} \otimes C(X)$ denote the $\mathrm{C}^{*}$-algebra formed by the completion of $\mathfrak{A} \odot C(X)$ with respect to a $\mathrm{C}^{*}$-norm. This $\mathrm{C}^{*}$-norm exists and is unique since $C(X)$ is commutative, which is why we do not decorate the tensor (see 6 , Propositions 2.4.2 and 3.6.12]). Note that $\mathfrak{A} \odot C(X)$ is dense in $\mathfrak{A} \otimes C(X)$ by definition.

Let $T: \mathfrak{A} \otimes C(X) \rightarrow C(X, \mathfrak{A})$ denote the canonical *-isomorphism for which on elementary tensors is given by $T(a \otimes f)=a \cdot f$, where $a \cdot f: x \in X \mapsto a \cdot f(x) \in \mathfrak{A}$ (see 25, Theorem 6.4.17]). Let $\mu$ be a pure state on $C(X, \mathfrak{A})$. Then $\mu \circ T$ is a pure state on $\mathfrak{A} \otimes C(X)$. Now, since $C(X)$ is commutative, there exists a pure state $\phi$ on $\mathfrak{A}$ and a pure state $\nu$ on $C(X)$ such that $\left.(\mu \circ T)\right|_{\mathfrak{A} \odot C(X)}=\phi \odot \nu$ by 6, Corollary 3.4.3], where $\phi \odot \nu$ is a complex-valued linear map on $\mathfrak{A} \odot C(X)$ given on elementary tensors by $(\phi \odot \nu)(a \otimes f)=\phi(a) \nu(f)$ (we do not distinguish elementary tensors with $\odot$ since these elements are in $\mathfrak{A} \odot C(X) \subseteq \mathfrak{A} \otimes C(X)$ by definition). However, as $\nu$ is a pure state on $C(X)$, there exists $x \in X$ such that $\nu=\delta_{x}$, where $\delta_{x}: f \in C(X) \mapsto f(x) \in \mathbb{C}$ is the Dirac point mass at $x$ (combine [10, Theorem VII.8.7] and [25, Theorem 5.1.6]). Thus $\left.(\mu \circ T)\right|_{\mathfrak{A} \odot C(X)}=\phi \odot \delta_{x}$. Hence

$$
\begin{aligned}
\mu(T(a \otimes f)) & =\mu \circ T(a \otimes f)=\left(\phi \odot \delta_{x}\right)(a \otimes f)=\phi(a) \delta_{x}(f) \\
& =\phi(a) f(x)=\phi(a \cdot f(x))=\phi(T(a \otimes f)(x))=\phi_{x}(T(a \otimes f))
\end{aligned}
$$

for all $a \in \mathfrak{A}, f \in C(X)$ since $a \otimes f \in \mathfrak{A} \odot C(X)$. By linearity, it is immediate that $\mu$ and $\phi_{x}$ agree on $T(\mathfrak{A} \odot C(X))$. However, as $T$ is a ${ }^{*}$-isomorphism, we see that $T(\mathfrak{A} \odot C(X))$ is dense in $C(X, \mathfrak{A})$, which implies that $\mu=\phi_{x}$ on $C(X, \mathfrak{A})$ by continuity of states. This completes the proof.

We can now combine these results to provide upper bounds for the diameter of the Monge-Kantorovich metric for our seminorms on $C(X, \mathfrak{A})$.

Proposition 2.9. Let $\left(X, \mathrm{~d}_{X}\right)$ be a compact metric space and $\mathfrak{A}$ be a unital $C^{*}$-algebra. Let $\varphi \in \mathscr{S}(C(X, \mathfrak{A}))$.

With notation from Definition 2.3, if $\|\cdot\|_{\mathrm{n}}$ is any norm on $\mathfrak{A}$ that is equivalent to the $C^{*}{ }_{-n o r m}\|\cdot\|_{\mathfrak{A}}$ so that there exist $M>0, N>0$ such that $M\|\cdot\|_{\mathrm{n}} \leqslant\|\cdot\|_{\mathfrak{A}} \leqslant N\|\cdot\|_{\mathrm{n}}$, then:

(1) (a) if $q=C(X)$, then $\operatorname{diam}\left(\mathscr{S}(C(X, \mathfrak{A})), \mathrm{mk}_{\mathrm{L}_{\mathrm{d}_{X}}^{(\mathrm{n}), q}}\right) \leqslant 2+N \cdot \operatorname{diam}\left(X, \mathrm{~d}_{X}\right)$

(b) if $\mathfrak{A}=\mathbb{C}$, then $\operatorname{diam}\left(\mathscr{S}(C(X, \mathfrak{A})), \mathrm{mk}_{\mathrm{L}_{\mathrm{d}_{X}}^{(\mathrm{n}), q}}\right) \leqslant N \cdot \operatorname{diam}\left(X, \mathrm{~d}_{X}\right)$;

(2) $q=\mathbb{C}$ or $q=\varphi$, then $\operatorname{diam}\left(\mathscr{S}(C(X, \mathfrak{A})), \mathrm{mk}_{\mathrm{L}_{\mathrm{d}_{X}}^{(\mathrm{n}), q}}\right) \leqslant 2$.

Proof. We begin by noting that as $\operatorname{dom}\left(\mathrm{L}_{\mathrm{d}_{X}}^{(\mathrm{n}), q}\right)$ is dense in $\mathfrak{s a}(C(X, \mathfrak{A}))$ by Lemma 2.6 $\mathrm{mk}_{\mathrm{L}_{\mathrm{d}_{X}}^{(\mathrm{n}), q}}$ is a metric on $\mathscr{S}(C(X, \mathfrak{A})$ ) (possibly taking value $+\infty)$ - see Remark 1.4

For (1)(a) and (1)(b), assume that $q=C(X)$. First, we establish (1)(a). Thus, let $\mu, \nu$ be pure states on $C(X, \mathfrak{A})$. By Lemma 2.8 there exist $x, y \in X$ and pure states $\phi, \psi$ on $\mathfrak{A}$ such that $\mu=\phi_{x}$ and $\nu=\psi_{y}$. Fix $a \in \operatorname{dom}\left(\mathrm{L}_{\mathrm{d}_{X}, q}^{(\mathrm{n}),}\right), \mathrm{L}_{\mathrm{d}_{X}}^{(\mathrm{n}), q}(a) \leqslant 1$. Let $\varepsilon>0$. Since $q=C(X)$, there exists $b \in C\left(X, \mathbb{C} 1_{\mathfrak{A}}\right)$ such that $\|a-b\|_{C(X, A)} \leqslant 1+\varepsilon$. Therefore, as 
$b(x) \in \mathbb{C} 1_{\mathfrak{A}}$, we have $\left|\phi_{x}(b)-\psi_{x}(b)\right|=|\phi(b(x))-\psi(b(x))|=0$ since $\phi, \psi$ are states on $\mathfrak{A}$ and agree on scalars of $\mathfrak{A}$. Therefore

$$
\begin{aligned}
\left|\phi_{x}(a)-\psi_{x}(a)\right| & =\left|\phi_{x}(a)-\phi_{x}(b)+\psi_{x}(b)-\psi_{x}(a)\right| \\
& \leqslant\left|\phi_{x}(a-b)\right|+\left|\psi_{x}(a-b)\right| \leqslant 2 \cdot\|a-b\|_{\mathfrak{A}} \leqslant 2(1+\varepsilon)=2+2 \varepsilon .
\end{aligned}
$$

Since $\varepsilon>0$ was arbitrary, $\left|\phi_{x}(a)-\psi_{x}(a)\right| \leqslant 2$. Next, since $\mathrm{L}_{\mathrm{d}_{X}}^{(\mathrm{n}), q}(a) \leqslant 1$, we gather:

$$
\begin{aligned}
\left|\psi_{x}(a)-\psi_{y}(a)\right| & =|\psi(a(x))-\psi(a(y))|=|\psi(a(x)-a(y))| \leqslant\|a(x)-a(y)\|_{\mathfrak{A}} \\
& \leqslant N \cdot\|a(x)-a(y)\|_{\mathrm{n}} \leqslant N \cdot \mathrm{d}_{X}(x, y) \leqslant N \cdot \operatorname{diam}\left(X, \mathrm{~d}_{X}\right),
\end{aligned}
$$

and $|\mu(a)-\nu(a)|=\left|\phi_{x}(a)-\psi_{y}(a)\right|$

$$
\leqslant\left|\phi_{x}(a)-\psi_{x}(a)\right|+\left|\psi_{x}(a)-\psi_{y}(a)\right| \leqslant 2+N \cdot \operatorname{diam}\left(X, \mathrm{~d}_{X}\right) .
$$

Since $a \in \operatorname{dom}\left(\mathrm{L}_{\mathrm{d}_{X}}^{(\mathrm{n}), q}\right), \mathrm{L}_{\mathrm{d}_{X}}^{(\mathrm{n}), q}(a) \leqslant 1$ was arbitrary, we see that $\mathrm{mk}_{\mathrm{L}_{\mathrm{d}_{X}}^{(\mathrm{n}), q}}(\mu, \nu) \leqslant$ $2+N \cdot \operatorname{diam}\left(X, \mathrm{~d}_{X}\right)$. Next, since $\mathrm{mk}_{\mathrm{L}_{\mathrm{d}_{X}}^{(\mathrm{n}), q}}$ is convex in each variable in the sense of $[28$, Definition 9.1] and the state space is the closed convex hull of the pure states by [25. Corollary 5.1.10], we have that (1)(a) is proven as $\mu, \nu$ were arbitrary pure states on $C(X, \mathfrak{A})$. For (1)(b), if $\mathfrak{A}=\mathbb{C}$, then note that $(2.3)$ would be 0 as there is only one state on $\mathbb{C}$. This then proves $(1)(b)$.

For (2), the case $q=\mathbb{C}$ is immediate from [27, Proposition 1.6] and from the inequality $\left\|a+\mathbb{C} 1_{\mathfrak{A}}\right\|_{\mathfrak{A} / \mathbb{C} 1_{\mathfrak{A}}} \leqslant \mathrm{L}(a)$ for all $a \in \mathfrak{s a}(\mathfrak{A})$.

For the case $q=\varphi$, the fact that

$$
\left\|a+\mathbb{C} 1_{C(X, \mathfrak{A})}\right\|_{C(X, \mathfrak{A}) / \mathbb{C} 1_{C(X, \mathfrak{A})}}=\inf _{b \in \mathbb{C} 1_{C(X, \mathfrak{A})}}\|a-b\|_{C(X, \mathfrak{A})} \leqslant\left\|a-\varphi(a) 1_{C(X, \mathfrak{A})}\right\|_{C(X, \mathfrak{A})}
$$

for all $a \in \mathfrak{s a}(\mathfrak{A})$ completes the proof again by the argument of case $q=\mathbb{C}$.

Now, we are prepared to prove our main result of this section, which is that the seminorms of Definition 2.3 will be quasi-Leibniz Lip-norms for $C(X, \mathfrak{A})$ if and only if $\mathfrak{A}$ is finite-dimensional, and we note that this is the first result that not only assumes finite-dimensionality of $\mathfrak{A}$, but also requires finite-dimensionality of $\mathfrak{A}$.

THEOREM 2.10. Let $\left(X, \mathrm{~d}_{X}\right)$ be a compact metric space and let $\mathfrak{A}$ be a unital $C^{*}$-algebra and let $\mu \in \mathscr{S}(C(X, \mathfrak{A}))$ be a state.

By using notation from Definition 2.3, the following three statements are equivalent:

(i) the pair $\left(C(X, \mathfrak{A}), \mathrm{L}_{\mathrm{d}_{X}}^{(\mathrm{n}), q}\right)$ is a quasi-Leibniz compact quantum metric space;

(ii) $\mathfrak{A}$ is finite-dimensional;

(iii) $C(X, \mathfrak{A})$ is a standard homogeneous $C^{*}$-algebra of Definition 2.1 .

Furthermore, if $\mathfrak{A}$ is finite-dimensional and $M>0, N>0$ such that $M \cdot\|\cdot\|_{\mathrm{n}} \leqslant$ $\|\cdot\|_{\mathfrak{A}} \leqslant N \cdot\|\cdot\|_{\mathrm{n}}$, then:

(1) if $q$ is either $C(X)$ or $\mathbb{C}$, then $\mathrm{L}_{\mathrm{d}_{X}}^{(\mathrm{n}), q}$ is a $(N / M, 0)$-quasi-Leibniz Lip-norm;

(2) if $q=\mu$, then $\mathrm{L}_{\mathrm{d}_{X}}^{(\mathrm{n}), q}$ is a $(\max \{N / M, 2\}, 0)$-quasi-Leibniz Lip-norm.

Proof. For (ii) $\Longrightarrow$ (i), assume that $\mathfrak{A}$ is finite-dimensional, we begin by showing that the pair $\left(C(X, \mathfrak{A}), \mathrm{L}_{\mathrm{d}_{X}}^{(\mathrm{n}), q}\right)$ is a quasi-Leibniz compact quantum metric space. By Proposition 2.4 and Lemma 2.6. all that remains is to show that the Monge-Kantorovich metric 
metrizes the weak* topology of the state space. Also, by Proposition 2.9 and equivalence (2) of Theorem 1.5 , we only need to verify that there exists some $D>0$ such that the set

$$
\mathcal{B}_{1, D}=\left\{a \in \mathfrak{s a}(C(X, \mathfrak{A})): \mathrm{L}_{\mathrm{d}_{X}}^{(\mathrm{n}), q}(a) \leqslant 1 \text { and }\|a\|_{C(X, \mathfrak{A})} \leqslant D\right\}
$$

is totally bounded.

Let $D \in(0, \infty)$. The set $\mathcal{B}_{1, D}$ is equicontinuous since $l_{\mathrm{d}_{X}}^{(\mathrm{n})}(a) \leqslant 1$ for all $a \in \mathcal{B}_{1, D}$ and $\|\cdot\|_{\mathrm{n}}$ is equivalent to $\|\cdot\|_{\mathfrak{A}}$ by finite-dimensionality. Next, $\left\{a(x) \in \mathfrak{A}: a \in \mathcal{B}_{1, D}\right\} \subseteq$ $\left\{b \in \mathfrak{A}:\|b\|_{\mathfrak{A}} \leqslant D\right\}$ for each $x \in X$. Since $\mathfrak{A}$ is finite-dimensional, the set $\{a(x) \in$ $\left.\mathfrak{A}: a \in \mathcal{B}_{1, D}\right\}$ is totally bounded for each $x \in X$. Therefore, by a generalization of the Arzelà-Ascoli Theorem [24, Theorem 7.47.1] and a characterization of the topology on $C(X, \mathfrak{A})[24$, Theorem 7.46 .7 and 7.46 .8$]$, the set $\mathcal{B}_{1, D}$ is totally bounded in $C(X, \mathfrak{A})$ since $X$ is compact. Thus, by Theorem 1.5 , this direction is complete.

For $(\mathrm{i}) \Longrightarrow(\mathrm{ii})$, assume that the pair $\left(C(X, \mathfrak{A}), \mathrm{L}_{\mathrm{d}_{X}}^{(\mathrm{n}), q}\right)$ is a quasi-Leibniz compact quantum metric space. By Theorem 1.5, the set $\mathcal{B}_{1, D}$ is totally bounded for some $D \in(0, \infty)$. Note that it follows that $\mathcal{B}_{E, F}$ is also totally bounded for all $E, F \in(0, \infty)$ by scaling, where $\mathcal{B}_{E, F}$ is defined similarly to $\mathcal{B}_{1, D}$. Now, the space of constant functions of $C(X, \mathfrak{A})$ denoted by $K(X, \mathfrak{A})$ is canonically *-isomorphic to $\mathfrak{A}$. Denote this *-isomorphism by

$$
\kappa: a \in \mathfrak{A} \longmapsto(x \mapsto a) \in K(X, \mathfrak{A}) .
$$

Assume that $a \in \mathfrak{A}$ and $\|a\|_{\mathfrak{A}} \leqslant 1$. Then, we have $\|\kappa(a)\|_{C(X, \mathfrak{A})} \leqslant 1$ and $l_{\mathrm{d}_{X}}^{(\mathrm{n})}(\kappa(a))=0$ since $\kappa(a)$ is constant. First consider when $q=C(X)$ or $q=\mathbb{C}$, then since quotient norms are bounded above by the norm that they are induced by, we have:

$$
\mathrm{L}_{\mathrm{d}_{X}}^{(\mathrm{n}), q}(\kappa(a)) \leqslant\|\kappa(a)\|_{C(X, \mathfrak{A})} \leqslant 1 .
$$

Therefore $\kappa(a) \in \mathcal{B}_{1,1}$. In particular,

$$
\kappa\left(\left\{a \in \mathfrak{A}:\|a\|_{\mathfrak{A}} \leqslant 1\right\}\right) \subseteq \mathcal{B}_{1,1},
$$

and thus $\kappa\left(\left\{a \in \mathfrak{A}:\|a\|_{\mathfrak{A}} \leqslant 1\right\}\right)$ is totally bounded, which implies that $\{a \in \mathfrak{A}$ : $\left.\|a\|_{\mathfrak{A}} \leqslant 1\right\}$ is totally bounded since $\kappa$ is a ${ }^{*}$-isomorphism. Now, if $q=\mu$, then we would have:

$$
\mathbf{L}_{\mathrm{d}_{X}}^{\mathfrak{A}}(\kappa(a))=\left\|\kappa(a)-\mu(\kappa(a)) 1_{C(X, \mathfrak{A})}\right\|_{C(X, \mathfrak{A})} \leqslant 2\|\kappa(a)\|_{C(X, \mathfrak{A})} \leqslant 2
$$

for $a \in \mathfrak{A}$ such that $\|a\|_{\mathfrak{A}} \leqslant 1$. Thus, since $\mathcal{B}_{2,1}$ is also totally bounded, the same argument shows that the unit ball of $\mathfrak{A}$ is totally bounded. Thus, in either case, the $\mathrm{C}^{*}$-algebra $\mathfrak{A}$ is finite-dimensional by $[33,1.9(d)]$. (ii) $\Longleftrightarrow$ (iii) is given by definition. Statements (1) and (2) immediately follow by Proposition 2.4 .

REMARK 2.11. Theorem 2.10 does not imply that there cannot be quantum metric structure on $C(X, \mathfrak{A})$ for $\mathfrak{A}$ infinite-dimensional. It simply implies that the family of seminorms of Definition 2.3 only provide quantum metric structure on $C(X, \mathfrak{A})$, when $\mathfrak{A}$ is finitedimensional, and this, in turn, strongly suggests that our seminorms are a natural choice for the quantum metric structure on $C(X, \mathfrak{A})$, when $\mathfrak{A}$ is finite-dimensional.

The following corollary focuses on a particular case of interest and a motivating idea for this paper. It shows that we can still recover the classical structure of $\left(C(X), \mathrm{L}_{\mathrm{d}_{X}}\right)$ 
within $\left(C(X, \mathfrak{A}), \mathrm{L}_{\mathrm{d}_{X}}^{(\mathrm{n}), q}\right)$ for any finite-dimensional $\mathrm{C}^{*}$-algebra $\mathfrak{A}$ and a particular choice of $\mathrm{n}$ and $q$.

Corollary 2.12. If $\left(X, \mathrm{~d}_{X}\right)$ is a compact metric space and $\mathfrak{A}$ is a finite-dimensional $C^{*}$-algebra, then with notation from Definition 2.3 , the pair $\left(C(X, \mathfrak{A}), \mathrm{L}_{\mathrm{d}_{X}}^{(\mathfrak{A}), C(X)}\right)$ is a Leibniz compact quantum metric space such that $\mathrm{L}_{\mathrm{d}_{X}}^{(2), C(X)}$ recovers the standard Lipschitz seminorm on $C(X)$ denoted by $\mathrm{L}_{\mathrm{d}_{X}}$ from the canonical *-isomorphism of $C(X)$ onto $C\left(X, \mathbb{C} 1_{\mathfrak{A}}\right)$ given by

$$
\mathfrak{c}_{X}: f \in C(X) \longmapsto\left(x \in X \mapsto f(x) 1_{\mathfrak{A}}\right) \in C\left(X, \mathbb{C} 1_{\mathfrak{A}}\right) .
$$

Hence, we have $\mathrm{L}_{\mathrm{d}_{X}}=\mathrm{L}_{\mathrm{d}_{X}}^{(\mathfrak{A}), C(X)} \circ \mathfrak{c}_{X}$, and thus

$$
\Lambda\left(\left(C(X), \mathrm{L}_{\mathrm{d}_{X}}\right),\left(C\left(X, \mathbb{C} 1_{\mathfrak{A}}\right), \mathrm{L}_{\mathrm{d}_{X}}^{(\mathfrak{A}), C(X)}\right)\right)=0 .
$$

In particular $\left(C(X), \mathrm{L}_{\mathrm{d}_{X}}\right)=\left(C(X, \mathfrak{A}), \mathrm{L}_{\mathrm{d}_{X}}^{(\mathfrak{A}), C(X)}\right)$, when $\mathfrak{A}=\mathbb{C}$.

Proof. Fix $f \in C(X)$, we have

$$
\begin{aligned}
\mathrm{L}_{\mathrm{d}_{X}}^{(\mathfrak{A}), C(X)}\left(\mathfrak{c}_{X}(f)\right) & =\max \left\{l_{\mathrm{d}_{X}}^{(\mathfrak{A})}\left(\mathfrak{c}_{X}(f)\right),\left\|\mathfrak{c}_{X}(f)+C\left(X, \mathbb{C} 1_{\mathfrak{A}}\right)\right\|_{C(X, \mathfrak{A}) / C\left(X, \mathbb{C}_{\mathfrak{A}}\right)}\right\} \\
& =\max \left\{l_{\mathrm{d}_{X}}^{(\mathfrak{A})}\left(\mathfrak{c}_{X}(f)\right), 0\right\} \\
& =\sup \left\{\frac{\left\|f(x) 1_{\mathfrak{A}}-f(y) 1_{\mathfrak{A}}\right\|_{\mathfrak{A}}}{\mathrm{d}_{X}(x, y)}: x, y \in X, x \neq y\right\} \\
& =\sup \left\{\frac{|f(x)-f(y)| \cdot\left\|1_{\mathfrak{A}}\right\|_{\mathfrak{A}}}{\mathrm{d}_{X}(x, y)}: x, y \in X, x \neq y\right\}=\mathrm{L}_{\mathrm{d}_{X}}(f) .
\end{aligned}
$$

This along with Theorem 2.10 completes the proof.

The last result of this section shows that when $\mathfrak{A}$ is finite-dimensional, then any two seminorms of Definition 2.3 are equivalent regardless of choice of $\mathrm{n}$ or $q$ (this is quite surprising since the quotient norms associated to $q=C(X)$ and $q=\mathbb{C}$ have different kernels on $C(X, \mathfrak{A})$ as long as $\mathfrak{A} ¥ \mathbb{C}$ and thus cannot be equivalent), and the map $\mathfrak{c}_{X}$ of the above corollary is bi-Lipschitz. Note that the following Proposition 2.14 rests mainly on a result of Latrémolière in [19]. First, a definition:

Definition 2.13. Let $\left(X, \mathrm{~d}_{X}\right)$ and $\left(Y, \mathrm{~d}_{Y}\right)$ be two pseudo-metric spaces, where pseudo means that distance 0 need not provide equal elements.

Fix $N>0$. A map $\pi:\left(X, \mathrm{~d}_{X}\right) \longrightarrow\left(Y, \mathrm{~d}_{Y}\right)$ is $N$-Lipschitz if $\mathrm{d}_{Y}(\pi(a), \pi(b)) \leqslant N$. $\mathrm{d}_{X}(a, b)$ for all $a, b \in X$.

The map $\pi$ is bi-Lipschitz, if furthermore, there exists $M>0$ such that $M \cdot \mathrm{d}_{X}(a, b) \leqslant$ $\mathrm{d}_{Y}(\pi(a), \pi(b)) \leqslant N \cdot \mathrm{d}_{X}(a, b)$ for all $a, b \in X$.

Proposition 2.14. Let $\left(X, \mathrm{~d}_{X}\right)$ be a compact metric space and $\mathfrak{A}$ be a finite-dimensional $C^{*}$-algebra. Let $\mu \in \mathscr{S}(C(X))$.

With notation from Definition 2.3, if $\|\cdot\|_{\mathrm{n}}$ and $\|\cdot\|_{\mathrm{m}}$ are norms on $\mathfrak{A}$ and $q$ and $p$ are either $C(X), \mathbb{C}$ or $\mu$, then the seminorms $\mathrm{L}_{\mathrm{d}_{X}}^{(\mathrm{n}), q}$ and $\mathrm{L}_{\mathrm{d}_{X}}^{(\mathrm{m}), p}$ are equivalent.

Furthermore, the map $\mathfrak{c}_{X}$ of Corollary 2.12 is bi-Lipschitz with respect to any $\mathrm{L}_{\mathrm{d}_{X}}^{(\mathrm{n}), q}$.

Proof. Since $\mathfrak{A}$ is finite-dimensional, the norms $\|\cdot\|_{\mathrm{n}}$ and $\|\cdot\|_{\mathrm{m}}$ are equivalent. Hence, we have $\operatorname{dom}\left(\mathrm{L}_{\mathrm{d}_{X}}^{(\mathrm{n}), q}\right)=\operatorname{dom}\left(\mathrm{L}_{\mathrm{d}_{X}}^{(\mathrm{m}), p}\right)$ since the terms determined by $q$ and $p$ are finite on 
all of $C(X, \mathfrak{A})$. Therefore, by Theorem 2.10 and 19 . Corollary 2.5], the seminorms $\mathrm{L}_{\mathrm{d}_{X}, q}^{(\mathrm{n})}$ and $L_{d_{X}}^{(m), p}$ are equivalent. The last statement of this proposition follows from this and Corollary 2.12 .

REMARK 2.15. Corollary 2.12 and Proposition 2.14 may suggest that the Lip-norm of Corollary 2.12 is the best choice from our family of seminorms for a $\operatorname{Lip}$-norm on $C(X, \mathfrak{A})$ for $X$ compact metric and $\mathfrak{A}$ finite-dimensional. However, we argue that it can depend on the situation. In particular, we use the flexibility on the choice of a norm on $\mathfrak{A}$ in Sections 3 and 4 to obtain positive results that may not be possible with the $\mathrm{C}^{*}$-norm on $\mathfrak{A}$.

In regards to why we consider other $q$ 's aside from $q=C(X)$, we first consider $q=\mathbb{C}$. Let $Y$ be a compact metric space and $\mathfrak{B}$ be a finite-dimensional $\mathrm{C}^{*}$-algebra. Note that any unital ${ }^{*}$-monomorphism $\pi: C(X, \mathfrak{A}) \longmapsto C(Y, \mathfrak{B})$, will be an isometry with respect to the quotient norms associated to $q=\mathbb{C}$ but not necessarily preserve the quotient norms associated to $q=C(X)$. So, if $q=C(X)$, then it would be much more difficult to verify if $\pi$ were contractive with respect to the Lip-norms of $C(X, \mathfrak{A})$ and $C(Y, \mathfrak{A})$ than in the case of $q=\mathbb{C}$. And, contractivity is vital to results pertaining to the Gromov-Hausdorff propinquity (see [29, Proposition 8.5] and [3. Theorem 3.5], for example). The reason to consider $q=\mu$ is similar to $q=\mathbb{C}$, except that a unital $*$-monomorphism need not preserves all states, but it does occur often enough - especially in the setting of inductive sequences and inductive limits - and the quantity given by $q=\mu$ in the Lip-norm can be much easier to calculate than a quotient norm.

3. Isometries from metrics into quantum metrics. In order for our seminorms on $C(X, \mathfrak{A})$ of the previous section to be a suitable generalization of the Lipschitz seminorm on $C(X)$, we claim that there should be a natural embedding of $X$ into the state space of $C(X, \mathfrak{A})$ which captures some of the metric structure of $X$ using the Monge-Kantorovich metric and not just the topological structure. This is because this happens in the classical case when $\mathfrak{A}=\mathbb{C}$ as discussed in the introduction. When $\mathfrak{A}$ is finite-dimensional, the purpose of this section is to verify that we can accomplish this claim for our entire family of seminorms of Definition 2.3 by providing a bi-Lipschitz embedding that can be strengthened to an isometry in many intuitive cases that are still valid for all finitedimensional $\mathfrak{A}$ and compact metric $X$.

For the remainder of this paper, we restrict our attention to finite-dimensional $\mathrm{C}^{*}$-algebras that are equal to finite direct sums of complex-valued matrix algebras. This is so that we can provide explicit estimates and the results of this section are still true for any finite-dimensional $\mathrm{C}^{*}$-algebra $\mathfrak{A}$ in $C(X, \mathfrak{A})$, which is explained in the following remark.

REMARK 3.1. Since every finite-dimensional $C^{*}$-algebra $\mathfrak{B}$ is ${ }^{*}$-isomorphic to a finite direct sums of complex-valued matrix algebras [11, Theorem III.1.1], we assume that every finite-dimensional $\mathrm{C}^{*}$-algebra is equal to $\bigoplus_{k=0}^{n} M_{m_{k}}(\mathbb{C})$ for some $n \in \mathbb{N}, m_{k} \in \mathbb{N} \backslash\{0\}$ for $k \in\{0, \ldots, n\}$ for the rest of this paper. This will cause no loss of generality for the results of this section. Indeed, let $\left(X, \mathrm{~d}_{X}\right)$ be a compact metric space, and assume 
$\pi: \mathfrak{B} \longrightarrow \bigoplus_{k=0}^{n} M_{m_{k}}(\mathbb{C})$ is a *-isomorphism, then the map

$$
\Pi: b \in C(X, \mathfrak{B}) \longmapsto(x \in X \mapsto \pi(b(x))) \in C\left(X, \bigoplus_{k=0}^{n} M_{m_{k}}(\mathbb{C})\right)
$$

is a unital *-isomorphism, and $\mathrm{L}_{\mathrm{d}_{X}}^{(\mathrm{n}), q} \circ \Pi$ would define a Lip-norm for $C(X, \mathfrak{B})$ with the same properties of $\mathrm{L}$, and furthermore, the dual map

$$
\Pi^{*}: \mu \in \mathscr{S}\left(C\left(X, \bigoplus_{k=0}^{n} M_{m_{k}}(\mathbb{C})\right)\right) \mapsto \mu \circ \Pi \in \mathscr{S}(C(X, \mathfrak{B}))
$$

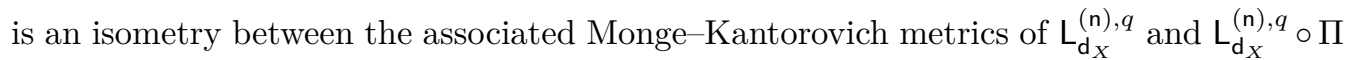
by [29, Thoerem 6.2].

Definition 3.2. Let $\mathfrak{A}=\bigoplus_{k=0}^{n} M_{m_{k}}(\mathbb{C})$ be a finite-dimensional $\mathrm{C}^{*}$-algebra for some $n \in \mathbb{N}$ and $m_{k} \in \mathbb{N} \backslash\{0\}$ for $k \in\{0, \ldots, n\}$. Fix $k \in\{0, \ldots, n\}$ and $p, q \in\left\{1, \ldots, m_{k}\right\}$. Let $e_{k,(p, q)} \in \mathfrak{A}$ denote the matrix unit such that $e_{k,(p, q)}=\left(a^{1}, \ldots, a^{n}\right) \in \mathfrak{A}$ and $a^{j}=0$ for $j \in\{0, \ldots, n\} \backslash\{k\}$ and $a^{k}$ is the matrix in $M_{m_{k}}(\mathbb{C})$ that is 1 in the $p$-row, $q$-column entry and 0 elsewhere. We will use $e_{k,(p, q)}$ to denote this element in $\mathfrak{A}$ as well as its projection onto $M_{m_{k}}(\mathbb{C})$, which is $a^{k}$.

Notation 3.3. Let $\left(X, \mathrm{~d}_{X}\right)$ be a compact metric space. Assume that $\mathfrak{A}$ is finite-dimensional and that there exist $n \in \mathbb{N}$ and $m_{k} \in \mathbb{N} \backslash\{0\}$ for $k \in\{0, \ldots, n\}$ such that $\mathfrak{A}=\bigoplus_{k=0}^{n} M_{m_{k}}(\mathbb{C})$. Let $\mathfrak{A} \otimes C(X)$ denote the $\mathrm{C}^{*}$-algebra formed over the algebraic tensor product of $\mathfrak{A}$ and $C(X)$. By [6, Propositions 2.4.2 and 3.6.12], we do not need to decorate the tensor (see the proof of Lemma 2.8 for details). The map

$$
\pi_{\otimes}^{\mathfrak{A}, X}: a \in C(X, \mathfrak{A}) \longmapsto \sum_{k=0}^{n} \sum_{p, q=1}^{m_{k}} e_{k,(p, q)} \otimes a_{p, q}^{k} \in \mathfrak{A} \otimes C(X)
$$

is the canonical *-isomorphism, where $a_{p, q}^{k} \in C(X)$ and for each $x \in X$, the element $a_{p, q}^{k}(x) \in \mathbb{C}$ is the projection of $a(x)$ onto the $p$-row, $q$-column coordinate of $M_{m_{k}}(\mathbb{C})$ (continuity of $a_{p, q}^{k}$ follows by finite-dimensionality and definition of product topology). That $\pi_{\otimes}^{\mathfrak{A}, X}$ is a ${ }^{*}$-isomorphism follows from the observation that it is the inverse of the map $T$ used in the proof of Lemma 2.8

For $\mu \in \mathscr{S}(\mathfrak{A}), \nu \in \mathscr{S}(C(X))$, let $\mu \otimes \nu: \mathfrak{A} \otimes C(X) \longrightarrow \mathbb{C}$ denote the state on $\mathfrak{A} \otimes C(X)$ such that on elementary tensors $(\mu \otimes \nu)(a \otimes f)=\mu(a) \nu(f)$ for all $a \in \mathfrak{A}$, $f \in C(X)$ 6, Proposition 3.4.6]. For $x \in X$, let

$$
\delta_{x}: f \in C(X) \longmapsto f(x) \in \mathbb{C}
$$

denote the Dirac point mass at $x$. The map $\delta_{x} \in \mathscr{S}(C(X))$, and for a state $\mu \in \mathscr{S}(\mathfrak{A})$, let

$\begin{array}{ll}\text { (1) } & k_{\mu}^{\mathfrak{A}}=\sum_{k=0}^{n} \sum_{p, q=1}^{m_{k}}\left|\mu\left(e_{k,(p, q)}\right)\right|, \\ \text { (2) } & \mu_{x}=\left(\mu \otimes \delta_{x}\right) \circ \pi_{\otimes}^{\mathfrak{A}, X} \in \mathscr{S}(C(X, \mathfrak{A})), \\ \text { (3) } & \Delta_{\mu}^{\mathfrak{A}}: x \in\left(X, \mathrm{~d}_{X}\right) \longmapsto \mu_{x} \in \mathscr{S}(C(X, \mathfrak{A})) .\end{array}$ 
Now, it is easy to show that the map $\Delta_{\mu}^{\mathfrak{A}}$ is a homeomorphism onto its image with respect to the weak* topology for any state $\mu \in \mathscr{S}(\mathfrak{A})$ (we, in fact, verify this in Proposition 3.6. However, when $X$ is metric we will show that $\Delta_{\mu}^{\mathfrak{A}}$ captures some metric structure when $\mu \in \mathscr{S}(\mathfrak{A})$ is any state by way of the Monge-Kantorovich metric (quantum metric) induced by the seminorms of Definition 2.3. Furthermore, for particular choices of states, we will be capable of isometrically embedding $X$ into $\mathscr{S}(C(X, \mathfrak{A}))$, which thus generalizes the classical case of $\mathfrak{A}=\mathbb{C}$ to the case when $\mathfrak{A}$ is finite-dimensional (the case of $\mathfrak{A}=\mathbb{C}$ was discussed in the introduction). Proposition 3.6 also shows that for any state $\mu$ and any of the seminorms of Definition 2.3, we can embed $X$ homeomorphically into $\mathscr{S}(C(X, \mathfrak{A}))$ with a $k$-Lipschitz map, where the constant $k$ depends on $\mu$, the matrix units of Definition 3.2 and the choice of norm on the finite-dimensional $\mathrm{C}^{*}$-algebra $\mathfrak{A}$. First, we state the following lemma about a certain standard norm on the underlying vector space of a finite-dimensional $\mathrm{C}^{*}$-algebra, which will prove useful later as well.

Lemma 3.4. Fix $n \in \mathbb{N}$ and $m_{k} \in \mathbb{N} \backslash\{0\}$ for $k \in\{0, \ldots, n\}$. Let $\mathfrak{A}=\bigoplus_{k=0}^{n} M_{m_{k}}(\mathbb{C})$ be a finite-dimensional $C^{*}$-algebra. Let $a=\left(a^{k}\right)_{k=0}^{n} \in \mathfrak{A}$, where $a^{k} \in M_{m_{k}}(\mathbb{C})$ for all $k \in\{0, \ldots, n\}$. Fix $k \in\{0, \ldots, n\}$ and let $a_{p, q}^{k}$ denote the p-row, q-column entry of the matrix $a^{k} \in M_{m_{k}}(\mathbb{C})$. Let $m_{\mathfrak{A}}=\max _{k \in\{0, \ldots, n\}} m_{k}$.

If $\|\cdot\|_{\infty, \mathfrak{A}}$ denotes the norm on $\mathfrak{A}$ defined by $\|a\|_{\infty, \mathfrak{A}}=\max _{k \in\{0, \ldots, n\}}\left\|a^{k}\right\|_{\infty}$, where $\left\|a^{k}\right\|_{\infty}=\max _{p, q \in\left\{1, \ldots, m_{k}\right\}}\left|\left(a^{k}\right)_{p, q}\right|$ for each $k \in\{0, \ldots, n\}$, then this norm is equivalent to $\|\cdot\|_{\mathfrak{A}}$ by

$$
\frac{1}{m_{\mathfrak{A}}}\|\cdot\|_{\mathfrak{A}} \leqslant\|\cdot\|_{\infty, \mathfrak{A}} \leqslant\|\cdot\|_{\mathfrak{A}} .
$$

Proof. The conclusion follows from the table of norm equivalences 14, page 314].

REMARK 3.5. If, in Corollary 2.12 we used the norm $\|\cdot\|_{\infty, \mathfrak{A}}$ of Lemma 3.4 instead of $\|\cdot\|_{\mathfrak{A}}$, then the map $\mathfrak{c}_{X}$ would still be an isometry of the Lip-norms $\mathrm{L}_{\mathrm{d}_{X}}$ and $\mathrm{L}_{\mathrm{d}_{X}}^{(\infty, \mathfrak{A}), C(X)}$ by the same argument of the proof of Corollary 2.12 since the norms $\|\cdot\|_{\infty, \mathfrak{A}}$ and $\|\cdot\|_{\mathfrak{A}}$ agree on scalars, but the associated compact quantum metric space would not be Leibniz but $\left(m_{\mathfrak{A}}, 0\right)$-quasi-Leibniz by Theorem 2.10 and Lemma 3.4

Proposition 3.6. Let $\left(X, \mathrm{~d}_{X}\right)$ be a compact metric space and let $\mathfrak{A}=\bigoplus_{k=0}^{n} M_{m_{k}}(\mathbb{C})$ be a finite-dimensional $C^{*}$-algebra, such that $n \in \mathbb{N}$ and $m_{k} \in \mathbb{N} \backslash\{0\}$ for $k \in\{0, \ldots, n\}$.

By (3.1), if $\mu \in \mathscr{S}(\mathfrak{A})$, then the map $\Delta_{\mu}^{\mathfrak{A}}$ is a homeomorphism onto its image with respect to the weak* topology on $\mathscr{S}(C(X, \mathfrak{A}))$.

Furthermore, using notation from Definitions 2.3 and 1.3 , if $\|\cdot\|_{\mathrm{n}}$ is a norm on $\mathfrak{A}$ and $M, N>0$ such that $M \cdot\|\cdot\|_{\mathrm{n}} \leqslant\|\cdot\|_{\mathfrak{A}} \leqslant N \cdot\|\cdot\|_{\mathrm{n}}$, then for all $x, y \in X$, we have

$$
\mathrm{mk}_{\mathrm{L}_{\mathrm{d}_{X}}^{(\mathrm{n}), q}}\left(\Delta_{\mu}^{\mathfrak{A}}(x), \Delta_{\mu}^{\mathfrak{A}}(y)\right) \leqslant N \cdot k_{\mu}^{\mathfrak{A}} \cdot \mathrm{d}_{X}(x, y),
$$

and thus $\Delta_{\mu}^{\mathfrak{A}}$ is $N \cdot k_{\mu}^{\mathfrak{A}}$-Lipschitz.

Proof. To show that $\Delta_{\mu}^{\mathfrak{A}}$ is a homeomorphism onto its image, we do not need to first show the Lipschitz condition and we do not need that $X$ is metric; however, we still rely on $X$ being Hausdorff. Let $\left(x_{\lambda}\right)_{\lambda \in \Lambda} \subseteq X$ be a net that converges to $x \in X$. Thus, for any 
$f \in C(X),\left(f\left(x_{\lambda}\right)_{\lambda \in \Lambda}\right) \subset \mathbb{C}$ converges to $f(x) \in \mathbb{C}$. Let $a \in C(X, \mathfrak{A})$ and fix $\lambda \in \Lambda$. Then

$$
\begin{aligned}
\Delta_{\mu}^{\mathfrak{A}}\left(x_{\lambda}\right)(a) & =\mu_{x_{\lambda}}(a)=\left(\mu \otimes \delta_{x_{\lambda}}\right)\left(\sum_{k=0}^{n} \sum_{p, q=1}^{m_{k}} e_{k,(p, q)} \otimes a_{p, q}^{k}\right) \\
& =\sum_{k=0}^{n} \sum_{p, q=1}^{m_{k}} \mu\left(e_{k,(p, q)}\right) \delta_{x_{\lambda}}\left(a_{p, q}^{k}\right)=\sum_{k=0}^{n} \sum_{p, q=1}^{m_{k}} \mu\left(e_{k,(p, q)}\right) a_{p, q}^{k}\left(x_{\lambda}\right) .
\end{aligned}
$$

Since this sum is finite and $\lambda \in \Lambda$ was arbitrary, we see that $\left(\Delta_{\mu}^{\mathfrak{A}}\left(x_{\lambda}\right)(a)\right)_{\lambda \in \Lambda} \subset \mathbb{C}$ converges to $\Delta_{\mu}^{\mathfrak{A}}(x)(a) \in \mathbb{C}$. Thus, the net $\left(\Delta_{\mu}^{\mathfrak{A}}\left(x_{\lambda}\right)\right)_{\lambda \in \Lambda} \subset \mathscr{S}(C(X, \mathfrak{A}))$ converges to $\Delta_{\mu}^{\mathfrak{A}}(x) \in \mathscr{S}(C(X, \mathfrak{A}))$ with respect to the weak* topology since $a \in C(X, \mathfrak{A})$ was arbitrary. Hence, since $X$ is compact and the weak* topology is Hausdorff, $\Delta_{\mu}^{\mathfrak{A}}$ is a homeomorphism onto its image.

Next, fix $x, y \in X$ and let $a \in \operatorname{dom}\left(\mathrm{L}_{\mathrm{d}_{X}}^{(\mathrm{n}, q}\right)$ such that $\mathrm{L}_{\mathrm{d}_{X}}^{(\mathrm{n}, q}(a) \leqslant 1$. Using Lemma 3.4 . we have:

$$
\frac{1}{N}\|a(x)-a(y)\|_{\infty, \mathfrak{A}} \leqslant \frac{1}{N}\|a(x)-a(y)\|_{\mathfrak{A}} \leqslant\|a(x)-a(y)\|_{\mathrm{n}} \leqslant \mathrm{d}_{X}(x, y),
$$

which provides that $\|a(x)-a(y)\|_{\infty, \mathfrak{A}} \leqslant N \mathrm{~d}_{X}(x, y)$. Hence, following (3.2), we gather:

$$
\begin{aligned}
& \left|\Delta_{\mu}^{\mathfrak{A}}(x)(a)-\Delta_{\mu}^{\mathfrak{A}}(y)(a)\right|=\left|\mu_{x}(a)-\mu_{y}(a)\right| \\
& =\left|\sum_{k=0}^{n} \sum_{p, q=1}^{m_{k}} \mu\left(e_{k,(p, q)}\right) a_{p, q}^{k}(x)-\sum_{k=0}^{n} \sum_{p, q=1}^{m_{k}} \mu\left(e_{k,(p, q)}\right) a_{p, q}^{k}(y)\right| \\
& \leqslant \sum_{k=0}^{n} \sum_{p, q=1}^{m_{k}}\left|\mu\left(e_{k,(p, q)}\right)\right|\left|a_{p, q}^{k}(x)-a_{p, q}^{k}(y)\right| \\
& \leqslant N\left(\sum_{k=0}^{n} \sum_{p, q=1}^{m_{k}}\left|\mu\left(e_{k,(p, q)}\right)\right|\right) \mathrm{d}_{X}(x, y)=N k_{\mu}^{\mathfrak{A}} \mathrm{d}_{X}(x, y),
\end{aligned}
$$

which implies that $\mathrm{mk}_{\mathrm{L}_{\mathrm{d}_{X}}^{(\mathrm{n}), q}}\left(\Delta_{\mu}^{\mathfrak{A}}(x), \Delta_{\mu}^{\mathfrak{A}}(y)\right) \leqslant N k_{\mu}^{\mathfrak{A}} \mathrm{d}_{X}(x, y)$ for all $x, y \in X$ by Definition 1.3 , and the proof is complete.

In order to produce bi-Lipschitz maps and isometries, we want to know more information about the expression $k_{\mu}^{\mathfrak{A}}$, which requires us to focus our attention on particular states. We will consider the family of tracial states of $\mathfrak{A}$. We note that the following theorem does include all tracial states on $\mathfrak{A}$ by [11, Example IV.5.4].

THEOREM 3.7. Let $\left(X, \mathrm{~d}_{X}\right)$ be a compact metric space and let $\mathfrak{A}=\bigoplus_{k=0}^{n} M_{m_{k}}(\mathbb{C})$ be a finite-dimensional $C^{*}$-algebra, such that $n \in \mathbb{N}$ and $m_{k} \in \mathbb{N} \backslash\{0\}$ for $k \in\{0, \ldots, n\}$. Let $\|\cdot\|_{\mathrm{n}}$ be a norm on $\mathfrak{A}$, let $M, N>0$ be such that $M \cdot\|\cdot\|_{\mathrm{n}} \leqslant\|\cdot\|_{\mathfrak{A}} \leqslant N \cdot\|\cdot\|_{\mathrm{n}}$ and let $\mu \in \mathscr{S}(C(X, \mathfrak{A}))$. Let $v=\left(v_{0}, \ldots, v_{n}\right) \in \mathbb{R}^{n+1}$ such that $v_{k} \in[0,1]$ for all $k \in\{0, \ldots, n\}$ and $1=\sum_{k=0}^{n} v_{k}$.

If we let $\operatorname{tr}_{v}^{\mathfrak{A}}=\bigoplus_{k=0}^{n} v_{k} \operatorname{tr}_{m_{k}}: \mathfrak{A} \longrightarrow \mathbb{C}$, where $\operatorname{tr}_{m_{k}}=\frac{1}{m_{k}} \operatorname{Tr}$ is the unique tracial state on $M_{m_{k}}(\mathbb{C})$ for all $k \in\{0, \ldots, n\}$, then using notation from Definitions 2.3, 1.3, and expression (3.1), we deduce that

$$
\Delta_{\operatorname{tr}_{v}^{\mathfrak{A}}}^{\mathfrak{A}}:\left(X, \mathrm{~d}_{X}\right) \longrightarrow\left(\mathscr{S}(C(X, \mathfrak{A})) \mathrm{mk}_{\mathrm{L}_{\mathrm{d}_{X}}^{(\mathrm{n}, q}}\right),
$$


is bi-Lipschitz, and in particular:

(1) if $q=C(X)$, then for all $x, y \in X$

$$
M \cdot \mathrm{d}_{X}(x, y) \leqslant \mathrm{mk}_{\mathrm{L}_{\mathrm{d}_{X}}^{(\mathrm{n}), C(X)}}\left(\Delta_{\operatorname{tr}_{v}^{\mathfrak{A}}}^{\mathfrak{A}}(x), \Delta_{\operatorname{tr}_{v}^{\mathfrak{A}}}^{\mathfrak{A}}(y)\right) \leqslant N \cdot \mathrm{d}_{X}(x, y) ;
$$

(2) if $q=\mathbb{C}$, then:

(a) if $M \cdot \operatorname{diam}\left(X, \mathrm{~d}_{X}\right) \leqslant 1$, then for all $x, y \in X$

$$
M \cdot \mathrm{d}_{X}(x, y) \leqslant \mathrm{mk}_{\mathrm{L}_{\mathrm{d}_{X}}^{(\mathrm{n}), \mathbb{C}}}\left(\Delta_{\operatorname{tr}_{v}^{\mathfrak{A}}}^{\mathfrak{A}}(x), \Delta_{\operatorname{tr}_{v}^{\mathfrak{A}}}^{\mathfrak{A}}(y)\right) \leqslant N \cdot \mathrm{d}_{X}(x, y) ;
$$

(b) if $M \cdot \operatorname{diam}\left(X, \mathrm{~d}_{X}\right)>1$, then for all $x, y \in X$

$$
\frac{1}{\operatorname{diam}\left(X, \mathrm{~d}_{X}\right)} \cdot \mathrm{d}_{X}(x, y) \leqslant \mathrm{mk}_{\mathrm{L}_{\mathrm{d}_{X}}^{(\mathrm{n}, \mathbb{C}}}\left(\Delta_{\operatorname{tr}_{v}^{\mathfrak{A}}}^{\mathfrak{A}}(x), \Delta_{\operatorname{tr}_{v}^{\mathfrak{A}}}^{\mathfrak{A}}(y)\right) \leqslant N \cdot \mathrm{d}_{X}(x, y) ;
$$

(3) if $\mu \in \mathscr{S}(C(X, \mathfrak{A}))$ and $q=\mu$, then:

(a) if $2 M \cdot \operatorname{diam}\left(X, \mathrm{~d}_{X}\right) \leqslant 1$, then for all $x, y \in X$

$$
M \cdot \mathrm{d}_{X}(x, y) \leqslant \mathrm{mk}_{\mathrm{L}_{\mathrm{d}_{X}}^{(\mathrm{n}, \mu}}\left(\Delta_{\operatorname{tr}_{v}^{\mathfrak{A}}}^{\mathfrak{A}}(x), \Delta_{\operatorname{tr}_{v}^{\mathfrak{A}}}^{\mathfrak{A}}(y)\right) \leqslant N \cdot \mathrm{d}_{X}(x, y)
$$

(b) if $2 M \cdot \operatorname{diam}\left(X, \mathrm{~d}_{X}\right)>1$, then for all $x, y \in X$

$$
\frac{1}{2 \cdot \operatorname{diam}\left(X, \mathrm{~d}_{X}\right)} \cdot \mathrm{d}_{X}(x, y) \leqslant \mathrm{mk}_{\mathrm{L}_{\mathrm{d} X}^{(\mathrm{n}), \mu}}\left(\Delta_{\operatorname{tr}_{v}^{\mathfrak{A}}}^{\mathfrak{A}}(x), \Delta_{\operatorname{tr}_{v}^{\mathfrak{A}}}^{\mathfrak{A}}(y)\right) \leqslant N \cdot \mathrm{d}_{X}(x, y) .
$$

Proof. We begin by calculating $k_{\operatorname{tr}_{v}^{\mathfrak{A}}}^{\mathfrak{A}}$, which is independent of the choice of $q$ :

$$
\begin{aligned}
k_{\operatorname{tr}_{v}^{\mathfrak{A}}}^{\mathfrak{A}} & =\sum_{k=0}^{n} \sum_{p, q=1}^{m_{k}}\left|\operatorname{tr}_{v}^{\mathfrak{A}}\left(e_{k,(p, q)}\right)\right|=\sum_{k=0}^{n} \frac{v_{k}}{m_{k}} \sum_{p, q=1}^{m_{k}}\left|\operatorname{tr}_{m_{k}}\left(e_{k,(p, q)}\right)\right| \\
& =\sum_{k=0}^{n} \frac{v_{k}}{m_{k}} \sum_{p, q=1, p=q}^{m_{k}} 1=\sum_{k=0}^{n} \frac{v_{k}}{m_{k}} m_{k}=\sum_{k=0}^{n} v_{k}=1
\end{aligned}
$$

by definition of the trace of a matrix $\operatorname{Tr}$ and matrix units (Definition 3.2), where in the second line we view each $e_{k,(p, q)}$ as an element of $M_{m_{k}}(\mathbb{C})$. This establishes the upper bounds for statements (1), (2) and (3).

For statement (1) and the lower bound, assume $q=C(X)$. Fix $x, y \in X$. Define the function

$$
y_{\mathrm{d}_{X}}: z \in X \longmapsto \mathrm{d}_{X}(y, z) \in \mathbb{R}
$$

and note that $y_{\mathrm{d}_{X}} \in \mathfrak{s a}(C(X))$. Next, define $Y_{\mathrm{d}_{X}}(z)=y_{\mathrm{d}_{X}}(z) 1_{\mathfrak{A}}$ for all $z \in X$, and thus $Y_{\mathrm{d}_{X}} \in \mathfrak{s a}\left(C\left(X, \mathbb{C} 1_{\mathfrak{A}}\right)\right)$. We thus have for $w, z \in X$ :

$$
\begin{array}{r}
\left\|M Y_{\mathrm{d}_{X}}(w)-M Y_{\mathrm{d}_{X}}(z)\right\|_{\mathrm{n}}=M\left\|Y_{\mathrm{d}_{X}}(w)-Y_{\mathrm{d}_{X}}(z)\right\|_{\mathrm{n}} \leqslant\left\|Y_{\mathrm{d}_{X}}(w)-Y_{\mathrm{d}_{X}}(z)\right\|_{\mathfrak{A}} \\
=\left|y_{\mathrm{d}_{X}}(w)-y_{\mathrm{d}_{X}}(z)\right|=\left|\mathrm{d}_{X}(y, w)-\mathrm{d}_{X}(y, z)\right| \leqslant \mathrm{d}_{X}(w, z) .
\end{array}
$$

Hence $M Y_{\mathrm{d}_{X}} \in \operatorname{dom}\left(\mathrm{L}_{\mathrm{d}_{X}}^{(\mathrm{n}, C(X)}\right)$ with $\mathrm{L}_{\mathrm{d}_{X}}^{(\mathrm{n}, C(X)}\left(M Y_{\mathrm{d}_{X}}\right) \leqslant 1$ since

$$
\left\|M Y_{\mathrm{d}_{X}}+C\left(X, \mathbb{C} 1_{\mathfrak{A}}\right)\right\|_{C(X, \mathfrak{A}) / C\left(X, \mathbb{C} 1_{\mathfrak{A}}\right)}=0
$$


by construction. From Notation 3.3 note further that $\pi_{\otimes}^{\mathfrak{A}, X}\left(Y_{\mathrm{d}_{X}}\right)=1_{\mathfrak{A}} \otimes y_{\mathrm{d}_{X}} \in \mathfrak{A} \otimes C(X)$. Therefore

$$
\begin{aligned}
& \left|\Delta_{\operatorname{tr}_{v}^{\mathfrak{A}}}^{\mathfrak{A}}(x)\left(M Y_{\mathrm{d}_{X}}\right)-\Delta_{\operatorname{tr}_{v}^{\mathfrak{A}}}^{\mathfrak{A}}(y)\left(M Y_{\mathrm{d}_{X}}\right)\right|=\left|\operatorname{tr}_{v x}^{\mathfrak{A}}\left(M Y_{\mathrm{d}_{X}}\right)-\operatorname{tr}_{v y}^{\mathfrak{A}}\left(M Y_{\mathrm{d}_{X}}\right)\right| \\
& \quad=M\left|\left(\operatorname{tr}_{v}^{\mathfrak{A}} \otimes \delta_{x}\right)\left(1_{\mathfrak{A}} \otimes y_{\mathrm{d}_{X}}\right)-\left(\operatorname{tr}_{v}^{\mathfrak{A}} \otimes \delta_{y}\right)\left(1_{\mathfrak{A}} \otimes y_{\mathrm{d}_{X}}\right)\right| \\
& \quad=M\left|\delta_{x}\left(y_{\mathrm{d}_{X}}\right)-\delta_{y}\left(y_{\mathrm{d}_{X}}\right)\right|=M\left|\mathrm{~d}_{X}(y, x)-\mathrm{d}_{X}(y, y)\right|=M \mathrm{~d}_{X}(x, y) .
\end{aligned}
$$

Thus $M \mathrm{~d}_{X}(x, y) \leqslant \mathrm{mk}_{\mathrm{L}_{\mathrm{d}_{X}}^{(\mathrm{n}, C(X)}}\left(\Delta_{\operatorname{tr}_{v}^{\mathfrak{A}}}^{\mathfrak{A}}(x), \Delta_{\operatorname{tr}_{v}^{\mathfrak{A}}}^{\mathfrak{A}}(y)\right)$ by Definition 1.3

For statement (2) and the lower bound, assume $q=\mathbb{C}$. First assume that $M \cdot \operatorname{diam}\left(X, \mathrm{~d}_{X}\right) \leqslant 1$. Fix $x, y \in X$. Now, consider $M Y_{\mathrm{d}_{X}}$, which still satisfies (3.4). However, in the quotient norm, we have

$$
\begin{aligned}
& \left\|M Y_{\mathrm{d}_{X}}+\mathbb{C} 1_{C(X, \mathfrak{A})}\right\|_{C(X, \mathfrak{A}) / \mathbb{C} 1_{C(X, \mathfrak{A})}} \leqslant\left\|M Y_{\mathrm{d}_{X}}-M \cdot \operatorname{diam}\left(X, \mathrm{~d}_{X}\right) 1_{C(X, \mathfrak{A})}\right\|_{C(X, \mathfrak{A})} \\
& \leqslant M \sup _{z \in X}\left\|\mathrm{~d}_{X}(y, z) 1_{\mathfrak{A}}-\operatorname{diam}\left(X, \mathrm{~d}_{X}\right) 1_{\mathfrak{A}}\right\|_{\mathfrak{A}} \leqslant M \sup _{z \in X}\left|\mathrm{~d}_{X}(y, z)-\operatorname{diam}\left(X, \mathrm{~d}_{X}\right)\right| \\
& =M \sup _{z \in X}\left(\operatorname{diam}\left(X, \mathrm{~d}_{X}\right)-\mathrm{d}_{X}(y, z)\right) \leqslant M \cdot \operatorname{diam}\left(X, \mathrm{~d}_{X}\right) \leqslant 1,
\end{aligned}
$$

since $\operatorname{diam}\left(X, \mathrm{~d}_{X}\right) \geqslant \mathrm{d}_{X}(y, z) \geqslant 0$ for all $y, z \in X$. Therefore, the assumption $\mathrm{L}_{\mathrm{d}_{X}}^{(\mathrm{n}, \mathbb{C}}\left(M Y_{\mathrm{d}_{X}}\right) \leqslant 1$ and the argument of expression 3.5 applies, which proves (a) of statement $(2)$.

For (b) of statement (2), assume that $M \cdot \operatorname{diam}\left(X, \mathrm{~d}_{X}\right)>1$. By (3.4) and since $\operatorname{diam}\left(X, \mathrm{~d}_{X}\right)>0$, we have for all $w, z \in X$ :

$$
\begin{aligned}
& \left\|\frac{1}{\operatorname{diam}\left(X, \mathrm{~d}_{X}\right)} Y_{\mathrm{d}_{X}}(w)-\frac{1}{\operatorname{diam}\left(X, \mathrm{~d}_{X}\right)} Y_{\mathrm{d}_{X}}(z)\right\|_{\mathrm{n}} \\
= & \frac{1}{M \cdot \operatorname{diam}\left(X, \mathrm{~d}_{X}\right)}\left\|M Y_{\mathrm{d}_{X}}(w)-M Y_{\mathrm{d}_{X}}(z)\right\|_{\mathrm{n}} \leqslant \frac{1}{M \cdot \operatorname{diam}\left(X, \mathrm{~d}_{X}\right)} \mathrm{d}_{X}(w, z)<\mathrm{d}_{X}(w, z) .
\end{aligned}
$$

By the same argument of 3.6 ,

$$
\left\|\frac{1}{\operatorname{diam}\left(X, \mathrm{~d}_{X}\right)} Y_{\mathrm{d}_{X}}+\mathbb{C} 1_{C(X, \mathfrak{A})}\right\|_{C(X, \mathfrak{A}) / \mathbb{C} 1_{C(X, \mathfrak{A})}} \leqslant 1 .
$$

Following the process of expression $(3.5),(2)(\mathrm{b})$ is proven. Statement (3) follows the same process as statement (2) along with the observation that for $y \in X$

$$
\begin{aligned}
\left\|Y_{\mathrm{d}_{X}}-\mu\left(Y_{\mathrm{d}_{X}}\right) 1_{C(X, \mathfrak{A})}\right\|_{C(X, \mathfrak{A})} & \leqslant 2\left\|Y_{\mathrm{d}_{X}}\right\|_{C(X, \mathfrak{A})}=2 \sup _{z \in X}\left\|\mathrm{~d}_{X}(y, z) 1_{\mathfrak{A}}\right\|_{\mathfrak{A}} \\
& =2 \sup _{z \in X}\left|\mathrm{~d}_{X}(y, z)\right| \leqslant 2 \cdot \operatorname{diam}\left(X, \mathrm{~d}_{X}\right),
\end{aligned}
$$

which completes the proof.

REMARK 3.8. In Theorem 3.7 and in statements (2) and (3), the diameter of the metric space $X$ appears. This is not too surprising because in Proposition 2.9 we do not see any relationship between the bound on the Monge-Kantorovich metric and the metric space $X$ in the cases of $q=\mathbb{C}$ and $q=\mu$. Thus, it makes sense for the diameter to appear in these cases in Theorem 3.7 to somewhat correct this discrepancy. 
In the next corollary, we will see that particular choices of norms on $\mathfrak{A}$ in Theorem 3.7 will provide us with isometries. Indeed:

COROllary 3.9. Let $\left(X, \mathrm{~d}_{X}\right)$ be a compact metric space and let $\mathfrak{A}$ be a finite-dimensional $C^{*}$-algebra such that $\mathfrak{A}=\bigoplus_{k=0}^{n} M_{m_{k}}(\mathbb{C})$, such that $n \in \mathbb{N}$ and $m_{k} \in \mathbb{N} \backslash\{0\}$ for $k \in$ $\{0, \ldots, n\}$. Let $\mu \in \mathscr{S}(C(X, \mathfrak{A}))$. Let $v=\left(v_{0}, \ldots, v_{n}\right) \in \mathbb{R}^{n+1}$ such that $v_{k} \in[0,1]$ for all $k \in\{0, \ldots, n\}$ and $1=\sum_{k=0}^{n} v_{k}$.

If $\|\cdot\|_{\mathrm{n}}=\|\cdot\|_{\mathfrak{A}}$ or $\|\cdot\|_{\mathrm{n}}=\|\cdot\|_{\infty, \mathfrak{A}}$ of Lemma 3.4, then using notation from Theorem 3.7. we see that:

(1) if $q=C(X)$, then $\Delta_{\operatorname{tr}_{v}^{\mathfrak{A}}}^{\mathfrak{A}}$ is an isometry;

(2) if $q=\mathbb{C}$, then:

(a) if $\operatorname{diam}\left(X, \mathrm{~d}_{X}\right) \leqslant 1$, then $\Delta_{\operatorname{tr}_{v}^{\mathfrak{A}}}^{\mathfrak{A}}$ is an isometry;

(b) if $\operatorname{diam}\left(X, \mathrm{~d}_{X}\right)>1$, then for all $x, y \in X$

$$
\frac{1}{\operatorname{diam}\left(X, \mathrm{~d}_{X}\right)} \cdot \mathrm{d}_{X}(x, y) \leqslant \mathrm{mk}_{\mathrm{L}_{\mathrm{d}_{X}}^{(\mathrm{n}), \mathbb{C}}}\left(\Delta_{\operatorname{tr}_{v}^{\mathfrak{A}}}^{\mathfrak{A}}(x), \Delta_{\operatorname{tr}_{v}^{\mathfrak{A}}}^{\mathfrak{A}}(y)\right) \leqslant \mathrm{d}_{X}(x, y) ;
$$

(3) if $\mu \in \mathscr{S}(C(X, \mathfrak{A}))$ and $q=\mu$, then:

(a) if $2 \cdot \operatorname{diam}\left(X, \mathrm{~d}_{X}\right) \leqslant 1$, then $\Delta_{\operatorname{tr}_{v}^{\mathfrak{A}}}^{\mathfrak{A}}$ is an isometry;

(b) if $2 \cdot \operatorname{diam}\left(X, \mathrm{~d}_{X}\right)>1$, then for all $x, y \in X$

$$
\frac{1}{2 \cdot \operatorname{diam}\left(X, \mathrm{~d}_{X}\right)} \cdot \mathrm{d}_{X}(x, y) \leqslant \mathrm{mk}_{\mathrm{L}_{\mathrm{d} X}^{(\mathrm{n}), \mu}}\left(\Delta_{\operatorname{tr}_{v}^{\mathfrak{A}}}^{\mathfrak{A}}(x), \Delta_{\operatorname{tr}_{v}^{\mathfrak{A}}}^{\mathfrak{A}}(y)\right) \leqslant \mathrm{d}_{X}(x, y) .
$$

In particular, if $X=\mathbb{T}$ is the circle as either a subset of $\mathbb{C}$ or quotient space of $[0,1]$ with their standard metrics, then $\Delta_{\operatorname{tr}_{v}^{\mathfrak{A}}}^{\mathfrak{A}}$ is an isometry when $q=C(\mathbb{T})$ or $q=\mathbb{C}$.

Proof. If $\|\cdot\|_{\mathrm{n}}=\|\cdot\|_{\mathfrak{A}}$, then the conclusions follow immediately from Theorem 3.7 The case of $\|\cdot\|_{\mathrm{n}}=\|\cdot\|_{\infty, \mathfrak{A}}$ follows from the same arguments in the proof of Theorem 3.7 along with the observation that as $Y_{\mathrm{d}_{X}}$ is scalar-valued:

$$
\left\|Y_{\mathrm{d}_{X}}(w)-Y_{\mathrm{d}_{X}}(z)\right\|_{\infty, \mathfrak{A}}=\left|y_{\mathrm{d}_{X}}(w)-y_{\mathrm{d}_{X}}(z)\right|=\left\|Y_{\mathrm{d}_{X}}(w)-Y_{\mathrm{d}_{X}}(z)\right\|_{\mathfrak{A}}
$$

for $y \in X$ and all $w, z \in X$.

\section{Convergence of standard homogeneous $\mathrm{C}^{*}$-algebras and finite-dimensional} approximations. By Theorem 1.9 the topology induced by the Gromov-Hausdorff distance GH on compact metric spaces homeomorphically embeds into the quantum Gromov-Hausdorff propinquity topology by the following class map:

$$
\Gamma:\left(X, \mathrm{~d}_{X}\right) \in(\mathrm{CMS}, \mathrm{GH}) \longmapsto\left(C(X), \mathrm{L}_{\mathrm{d}_{X}} \in(\mathrm{qLCQMS}, \Lambda),\right.
$$

where CMS is the class of compact metric spaces and qLCQMS is the class of all quasiLeibniz compact quantum metric spaces. The bijection is with respect to the equivalence relations of isometry on the domain and quantum isometry on the codomain. However, this result was true with Rieffel's quantum distance dist ${ }_{q}$ in 2000 29, combine Proposition 4.7, Corollary 7.10, and Theorem 13.16]. And, since 2000, the question of whether the continuity of $\Gamma$ extends to matrices over $C(X)$ has been left unanswered. More formally, 
this question asks if for all $n \in \mathbb{N} \backslash\{0\}$, there exist Lip-norms $\mathrm{L}_{X}^{n}$ such that the map

$$
\Gamma^{M_{n}}:\left(X, \mathrm{~d}_{X}\right) \in(\mathrm{CMS}, \mathrm{GH}) \longmapsto\left(M_{n}(C(X)), \mathrm{L}_{X}^{n}\right) \in(\mathrm{qLCQMS}, \Lambda)
$$

is continuous. To be clear, this question involves not just continuity, but also asks what the Lip-norms $L_{X}^{n}$ should be. In this section, we finally answer this question by presenting Lip-norms from our work in previous sections for which continuity of this map does hold. This will also produce finite-dimensional approximations for $M_{n}(C(X))$ as every compact metric space may be approximated in the Hausdorff distance by finite subsets. We note that in this section we prove the continuity of the map in 4.1 in slightly more generality and for spaces of the form $C(X, \mathfrak{A})$, where $\left(X, \mathrm{~d}_{X}\right)$ is any compact metric and $\mathfrak{A}$ is a fixed finite-dimensional $\mathrm{C}^{*}$-algebra and note that $C\left(X, M_{n}(\mathbb{C})\right)$ is canonically ${ }^{*}$-isomorphic to $M_{n}(C(X))$.

The idea of our proof relies on a result of E. J. McShane [23, Theorem 1], which informally states that one can extend a real-valued $K$-Lipschitz map defined on a subset of a metric space to a $K$-Lipschitz map on the whole space. The real-valued condition is key as this is not true in general for complex valued functions (see [34, Example 1.5.7]. McShane's Theorem was used successfully by Latrémolière in [20, Theorem 6.6] along with the fact that Lip-norms need only be defined on self-adjoints (real-valued functions in this case) to show continuity of $\Gamma$. However, for $M_{n}(\mathbb{C})$ with $n \geqslant 2$, the self-adjoint elements may have complex entries. This has been the obstruction that has made this problem difficult to solve. Our solution is provided by the simple observation that Lipnorms are seminorms defined on a subspace of the $\mathbb{R}$-vector space of self-adjoint elements of a $\mathrm{C}^{*}$-algebra. We now define the seminorms which will provide our convergence results of this section, which are similar to the seminorms of (1) of Definition 2.3

Notation 4.1. Let $\left(X, \mathrm{~d}_{X}\right)$ be a compact metric space and $\mathfrak{A}$ be a finite-dimensional $\mathrm{C}^{*}$-algebra such that there exists $n \in \mathbb{N}$ and $m_{l} \in \mathbb{N} \backslash\{0\}$ for $l \in\{0, \ldots, n\}$ with $\mathfrak{A}=\bigoplus_{l=0}^{n} M_{m_{l}}(\mathbb{C})$.

For $\lambda \in \mathbb{C}$, let $\mathfrak{R e}(\lambda)$ denote its real part and $\mathfrak{I m}(\lambda)$ its imaginary part. Define $|\lambda|_{\infty}=\max \{|\mathfrak{R e}(\lambda)|,|\mathfrak{I m}(\lambda)|\}$, which defines an $\mathbb{R}$-norm over $\mathbb{C}$, and we note that $|\lambda|_{\infty} \leqslant|\lambda| \leqslant \sqrt{2}|\lambda|_{\infty}$ for all $\lambda \in \mathbb{C}$. For $a=\left(a_{j, k}^{l}\right)_{l \in\{0, \ldots, n\}, j, k \in\left\{1, \ldots, m_{l}\right\}} \in \mathfrak{s a}(\mathfrak{A})$, where $\left(a_{j, k}^{l}\right)_{j, k \in\left\{1, \ldots, m_{l}\right\}} \in M_{m_{l}}(\mathbb{C})$ for all $l \in\{0, \ldots, n\}$, define

$$
\|a\|_{\infty, \mathfrak{A}_{\mathbb{R}}}=\max _{l \in\{0, \ldots, n\}, j, k \in\left\{1, \ldots, m_{l}\right\}}\left|a_{j, k}^{l}\right|_{\infty}
$$

which is a norm over $\mathfrak{s a}(\mathfrak{A})$, but is not a norm over $\mathfrak{A}$ with respect to $\mathbb{C}$.

Define $m_{\mathfrak{A}}=\max _{l \in\{0, \ldots, n\}} m_{l}$. By Lemma 3.4 .

$$
\|a\|_{\infty, \mathfrak{A}_{\mathbb{R}}} \leqslant\|a\|_{\mathfrak{A}} \leqslant \sqrt{2} \cdot m_{\mathfrak{A}} \cdot\|a\|_{\infty, \mathfrak{A}_{\mathbb{R}}}
$$

for all $a \in \mathfrak{A}$. For all $a \in \mathfrak{s a}(C(X, \mathfrak{A}))$, using notation from Definition 2.3 define

$$
\mathrm{L}_{\mathrm{d}_{X}}^{\left(\infty, \mathfrak{A}_{\mathbb{R}}\right)}(a)=\max \left\{l_{\mathrm{d}_{X}}^{\left(\infty, \mathfrak{A}_{\mathbb{R}}\right)}(a), \inf _{r \in \mathbb{R}} \sup _{x \in X}\left\|a(x)-r 1_{\mathfrak{A}}\right\|_{\infty, \mathfrak{A}_{\mathbb{R}}}\right\} .
$$

We note that the idea to circumvent the difficulties of McShane's Theorem 23, Theorem 1] by utilizing the real and imaginary parts of complex numbers has appeared in the literature before and in particular in work of Latrémolière in [22, Remark 3.13]. 
Using the previous section, we summarize some of the quantum metric structure associated to the Lip-norm of the above definition.

Proposition 4.2. Let $\left(X, \mathrm{~d}_{X}\right)$ be a compact metric space and $\mathfrak{A}$ be a finite-dimensional $C^{*}$-algebra such that there exist $n \in \mathbb{N}$ and $m_{l} \in \mathbb{N} \backslash\{0\}$ for $l \in\{0, \ldots, n\}$ with $\mathfrak{A}=$ $\bigoplus_{l=0}^{n} M_{m_{l}}(\mathbb{C})$. Let $m_{\mathfrak{A}}=\max _{l \in\{0, \ldots, n\}} m_{l}$.

Using Notation 4.1, we see that $\left(C(X, \mathfrak{A}), \mathrm{L}_{\mathrm{d}_{X}}^{\left(\infty, \mathfrak{A}_{\mathbb{R}}\right)}\right)$ is a $\left(\sqrt{2} \cdot m_{\mathfrak{A}}, 0\right)$-quasi-Leibniz compact quantum metric space for which

$$
\operatorname{diam}\left(\mathscr{S}(C(X, \mathfrak{A})), \mathrm{mk}_{\mathrm{L}_{\mathrm{d}_{X}}^{\left(\infty, \mathfrak{A}_{\mathbb{R}}\right)}}\right) \leqslant 2 \sqrt{2} \cdot m_{\mathfrak{A}} .
$$

Proof. First, although $\|\cdot\|_{\infty, \mathfrak{A}_{\mathbb{R}}}$ is only a norm on $\mathfrak{s a}(\mathfrak{A})$, we note that the results from Section 2 used in this proof are valid for $\|\cdot\|_{\infty, \mathfrak{A}_{\mathbb{R}}}$ since $\|\cdot\|_{\infty, \mathfrak{A}_{\mathbb{R}}}$ satisfies $(4.2)$ on $\mathfrak{A}$.

For the quasi-Leibniz condition, we first note that $\mathbb{C} 1_{C(X, \mathfrak{A})}$ satisfies best approximation in $C(X, \mathfrak{A})$ since $\mathbb{C} 1_{C(X, \mathfrak{A})}$ is finite-dimensional. That is, if $a \in \mathfrak{s a}(\mathfrak{A})$, there exists

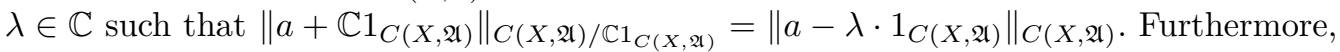
since $a \in \mathfrak{s a}(\mathfrak{A})$, we can assume that $\lambda \in \mathbb{R}$ since $\frac{\lambda+\lambda^{*}}{2}$ is also a best approximation. In

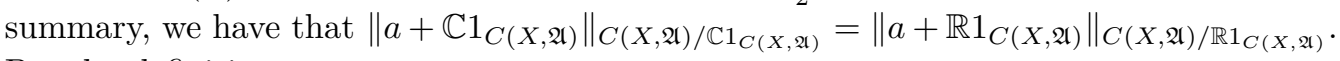
But, by definition,

$$
\left\|a+\mathbb{C} 1_{C(X, \mathfrak{A})}\right\|_{C(X, \mathfrak{A}) / \mathbb{C} 1_{C(X, \mathfrak{A})}}=\inf _{r \in \mathbb{R}}\left\|a-r 1_{C(X, \mathfrak{A})}\right\|_{C(X, \mathfrak{A})}=\inf _{r \in \mathbb{R}} \sup _{x \in X}\left\|a(x)-r 1_{\mathfrak{A}}\right\|_{\mathfrak{A}} .
$$

Hence, by 4.2, we have the equivalence

$$
\begin{aligned}
\inf _{r \in \mathbb{R}} \sup _{x \in X}\left\|(\cdot)(x)-r 1_{\mathfrak{A}}\right\|_{\infty, \mathfrak{A}_{\mathbb{R}}} & \leqslant\left\|(\cdot)+\mathbb{C} 1_{C(X, \mathfrak{A})}\right\| C(X, \mathfrak{A}) / \mathbb{C} 1_{C(X, \mathfrak{A})} \\
& \leqslant \sqrt{2} \cdot m_{\mathfrak{A}} \cdot \inf _{r \in \mathbb{R}} \sup _{x \in X}\left\|(\cdot)(x)-r 1_{\mathfrak{A}}\right\|_{\infty, \mathfrak{A}_{\mathbb{R}}} .
\end{aligned}
$$

However, as $\left\|(\cdot)+\mathbb{C} 1_{C(X, \mathfrak{A})}\right\|_{C(X, \mathfrak{A}) / \mathbb{C} 1_{C(X, \mathfrak{A})}}$ is $(1,0)$-quasi-Leibniz by [31, Theorem 3.1], then $\inf _{r \in \mathbb{R}} \sup _{x \in X}\left\|(\cdot)(x)-r 1_{\mathfrak{A}}\right\|_{\infty, \mathfrak{A}_{\mathbb{R}}}$ is $\left(\sqrt{2} \cdot m_{\mathfrak{A}}, 0\right)$-quasi-Leibniz by the same argument of Proposition 2.4 which provides the desired quasi-Leibniz condition for $L_{d_{X}}^{\left(\infty, \mathfrak{A}_{\mathbb{R}}\right)}$.

Next, we note that the expression $\inf _{r \in \mathbb{R}} \sup _{x \in X}\left\|(\cdot)(x)-r 1_{\mathfrak{A}}\right\|_{\infty, \mathfrak{A}_{\mathbb{R}}}$ is simply the quotient norm onto the scalars of $C(X, \mathfrak{s a}(\mathfrak{A})$ equipped with the supremum norm induced by $\|\cdot\|_{\infty, \mathfrak{A}_{\mathbb{R}}}$, i.e. $\sup _{x \in X}\|a(x)\|_{\infty, \mathfrak{A}_{\mathbb{R}}}$ for all $a \in C(X, \mathfrak{s a}(\mathfrak{A}))$. Thus, as $\mathbb{R} 1_{C(X, \mathfrak{A})}$ is a closed subspace of the Banach space $C(X, \mathfrak{s a}(\mathfrak{A}))$ with respect to this norm, then the kernel of $\mathrm{L}_{\mathrm{d}_{X}}^{\left(\infty, \mathfrak{A}_{\mathbb{R}}\right)}$ is $\mathbb{R} 1_{C(X, \mathfrak{A})}$. The domain of $\mathrm{L}_{\mathrm{d}_{X}}^{\left(\infty, \mathfrak{A}_{\mathbb{R}}\right)}$ is dense by Lemma 2.6. Expression (4.3) produces the bound on the diameter of the Monge-Kantorovich metric by 27 , Proposition 1.6]. Thus, the fact that $\left(C(X, \mathfrak{A}), \mathrm{L}_{\mathrm{d}_{X}}^{\left(\infty, \mathfrak{A}_{\mathbb{R}}\right)}\right)$ is a $\left(\sqrt{2} \cdot m_{\mathfrak{A}}, 0\right)$-quasi-Leibniz compact quantum metric space follows from Theorem 2.10

REMARK 4.3. Similar conclusions to Proposition 2.14 and the results of Section 3 can be made with respect to the Lip-norm $L_{d_{X}}^{\left(\infty, \mathfrak{A}_{\mathbb{R}}\right)}$, but we do not need them here and do not list them for the purpose of presentation.

We are ready to prove one of our main convergence results, which will show that our Lip-norm capitalizes on the real structure of $\mathfrak{s a}(\mathfrak{A})$ in more ways than one. Indeed, the second quantity in the expression of $L_{d_{X}}^{\left(\infty, \mathfrak{A}_{\mathbb{R}}\right)}$ pertains to bounds on elements in 
$\mathfrak{s a}(C(X, \mathfrak{A}))$. In particular, we require a more powerful version of McShane's theorem in that we need our Lipschitz extensions to preserve upper and lower bounds as well.

THEOREM 4.4. Let $\mathfrak{A}$ be a finite-dimensional $C^{*}$-algebra such that there exists $n \in \mathbb{N}$ and $m_{l} \in \mathbb{N} \backslash\{0\}$ for $l \in\{0, \ldots, n\}$ with $\mathfrak{A}=\bigoplus_{l=0}^{n} M_{m_{l}}(\mathbb{C})$. Let $m_{\mathfrak{A}}=\max _{l \in\{0, \ldots, n\}} m_{l}$.

If we use Notation 4.1, Theorem 4.2, and Convention 1.8, the class map

$$
\Gamma^{\mathfrak{A}}:\left(X, \mathrm{~d}_{X}\right) \in\left(\mathrm{CMS}, \mathrm{GH} \mapsto\left(C(X, \mathfrak{A}), \mathrm{L}_{\mathrm{d}_{X}}^{\left(\infty, \mathfrak{A}_{\mathbb{R}}\right)}\right) \in(\mathrm{qLCQMS}, \Lambda)\right.
$$

is well-defined and continuous.

In particular, we have for any compact metric spaces $\left(X, \mathrm{~d}_{X}\right),\left(Y, \mathrm{~d}_{Y}\right)$ :

$$
\Lambda\left(\left(C(X, \mathfrak{A}), \mathrm{L}_{\left.\mathrm{d}_{X}, \mathfrak{A}_{\mathbb{R}}\right)}^{\left({ }^{\prime}\right.}\right),\left(C(Y, \mathfrak{A}), \mathrm{L}_{\mathrm{d}_{Y}}^{\left(\infty, \mathfrak{A}_{\mathbb{R}}\right)}\right)\right) \leqslant \sqrt{2} \cdot m_{\mathfrak{A}} \cdot \mathrm{GH}\left(\left(X, \mathrm{~d}_{X}\right),\left(Y, \mathrm{~d}_{Y}\right)\right) .
$$

Proof. Proving inequality 4.4 would prove both well-defined and continuity of $\Gamma^{\mathfrak{A}}$ with respect to the stated equivalence relations of Convention 1.8. This proof follows the general strategy of the proof of $[20$, Theorem 6.6].

Let $\delta_{X, Y}=\mathrm{GH}\left(\left(X, \mathrm{~d}_{X}\right),\left(Y, \mathrm{~d}_{Y}\right)\right) \geqslant 0$. Let $\varepsilon>0$. By definition of the GromovHausdorff distance [7, Definition 7.3.10], there exists a metric space $\left(Z, \mathrm{~d}_{Z}\right)$ and isometries (not necessarily surjective) $f_{X}:\left(X, \mathrm{~d}_{X}\right) \rightarrow\left(Z, \mathrm{~d}_{Z}\right)$ and $f_{Y}:\left(Y, \mathrm{~d}_{Y}\right) \rightarrow\left(Z, \mathrm{~d}_{Z}\right)$ such that the Hausdorff distance Haus $_{\mathrm{d}_{Z}}\left(f_{X}(X), f_{Y}(Y)\right) \leqslant \delta_{X, Y}+\frac{\varepsilon}{8 \sqrt{2} \cdot m_{\mathfrak{A}}}$. Now, define a metric $\mathrm{d}_{X \sqcup Y}$ on the disjoint union $X \sqcup Y$ by:

$$
\mathrm{d}_{X \sqcup Y}(\alpha, \beta)= \begin{cases}\mathrm{d}_{X}(\alpha, \beta), & \text { if } \alpha, \beta \in X, \\ \mathrm{~d}_{Z}\left(f_{X}(\alpha), f_{Y}(\beta)\right)+\frac{\varepsilon}{8 \sqrt{2} \cdot m_{\mathfrak{A}}}, & \text { if } \alpha \in X, \beta \in Y, \\ \mathrm{~d}_{Z}\left(f_{X}(\beta), f_{Y}(\alpha)\right)+\frac{\varepsilon}{8 \sqrt{2} \cdot m_{\mathfrak{A}}}, & \text { if } \alpha \in Y, \beta \in X, \\ \mathrm{~d}_{Y}(\alpha, \beta), & \text { if } \alpha, \beta \in Y .\end{cases}
$$

Clearly $X$ and $Y$ embed isometrically into $\left(X \sqcup Y, \mathrm{~d}_{X \sqcup Y}\right)$ with respect to their associated metrics and

$$
\text { Haus }_{\mathrm{d}_{X} \sqcup Y}(X, Y) \leqslant \delta_{X, Y}+\frac{\varepsilon}{4 \sqrt{2} \cdot m_{\mathfrak{A}}}
$$

by [7, Remark 7.3.2], where $X, Y$ are viewed as subspaces of $X \sqcup Y$.

Define $W=\left\{(x, y) \in X \times Y: \mathrm{d}_{X \sqcup Y}(x, y) \leqslant \delta_{X, Y}+\frac{\varepsilon}{2 \sqrt{2} \cdot m_{\mathfrak{A}}}\right\}$. By construction, $W$ is compact in the product topology on $X \times Y$, and thus we equip $W$ with this topology. Now, fix $x \in X$. By definition of the Hausdorff distance and expression (4.5), there exists $y \in Y$ such that $\mathrm{d}_{X \sqcup Y}(x, y) \leqslant \delta_{X, Y}+\frac{\varepsilon}{4 \sqrt{2} \cdot m_{\mathfrak{A}}}+\frac{\varepsilon}{4 \sqrt{2} \cdot m_{\mathfrak{A}}}=\delta_{X, Y}+\frac{\varepsilon}{2 \sqrt{2} \cdot m_{\mathfrak{A}}}$. Thus, for every $x \in X$, there exists $y \in Y$ such that $(x, y) \in W$. Similarly, for every $y \in Y$, there exists $x \in X$ such that $(x, y) \in W$. In particular, the canonical projections $\rho_{X}$ : $(x, y) \in W \mapsto x \in X$ and $\rho_{Y}:(x, y) \in W \mapsto x \in Y$ are surjections and are continuous by definition of the product topology. Therefore, the two maps $\pi_{X}: f \in C(X) \mapsto$ $f \circ \rho_{X} \in C(W)$ and $\pi_{Y}: f \in C(Y) \mapsto f \circ \rho_{Y} \in C(W)$ are unital *-monomorphisms. Hence, we define a unital ${ }^{*}$-monomorphism $\pi_{X}^{\mathfrak{A}}: C(X, \mathfrak{A}) \rightarrow C(W, \mathfrak{A})$, where for every $a=\left(a_{j, k}^{l}\right)_{l \in\{0, \ldots, n\}, j, k \in\left\{1, \ldots, m_{l}\right\}} \in C(X, \mathfrak{A})$

$$
\pi_{X}^{\mathfrak{A}}\left(\left(a_{j, k}^{l}\right)_{l \in\{0, \ldots, n\}, j, k \in\left\{1, \ldots, m_{l}\right\}}\right)=\left(\pi_{X} \circ a_{j, k}^{l}\right)_{l \in\{0, \ldots, n\}, j, k \in\left\{1, \ldots, m_{l}\right\}}
$$


and $\pi_{Y}^{\mathfrak{A}}: C(Y, \mathfrak{A}) \rightarrow C(W, \mathfrak{A})$ is defined in the same way. Therefore, the tuple $\gamma_{X, Y, \varepsilon}=$ $\left(C(W, \mathfrak{A}), 1_{C(W, \mathfrak{A})}, \pi_{X}^{\mathfrak{A}}, \pi_{Y}^{\mathfrak{A}}\right)$ defines a bridge from $C(X, \mathfrak{A})$ to $C(Y, \mathfrak{A})$ by 20 , Definition 3.6]. We claim that this bridge's length [20, Definition 3.17] is less than or equal to $\sqrt{2} \cdot m_{\mathfrak{A}} \cdot \delta_{X, Y}+\frac{\varepsilon}{2}$. First, we note that the bridge's height [20, Definition 3.16] is 0 since the pivot is the identity $1_{C(W, \mathfrak{A})}$. Thus, we are left to find an upper bound for the bridge's reach [20, Definition 3.14].

Thus, let $a=\left(a_{j, k}^{l}\right)_{l \in\{0, \ldots, n\}, j, k \in\left\{1, \ldots, m_{l}\right\}} \in \mathfrak{s a}(C(X, \mathfrak{A}))$ such that $\mathrm{L}_{\mathrm{d}_{X}}^{\left(\infty, \mathfrak{A}_{\mathbb{R}}\right)}(a) \leqslant 1$. First, this implies that $\mathrm{L}_{\mathrm{d}_{X}}\left(\mathfrak{R e}\left(a_{j, k}^{l}\right)\right) \leqslant 1$ and $\mathrm{L}_{\mathrm{d}_{X}}\left(\mathfrak{I m}\left(a_{j, k}^{l}\right)\right) \leqslant 1$ for all $l \in\{0, \ldots, n\}$, $j, k \in\left\{1, \ldots, m_{l}\right\}$. For all $l \in\{0, \ldots, n\}, j, k \in\left\{1, \ldots, m_{l}\right\}$, let

$$
\widehat{\mathfrak{R e}\left(a_{j, k}^{l}\right)}, \widehat{\mathfrak{I m}\left(a_{j, k}^{l}\right)}: X \sqcup Y \rightarrow \mathbb{R}
$$

denote the Lipschitz-constant preserving extensions of $\mathfrak{R e}\left(a_{j, k}^{l}\right), \mathfrak{I m}\left(a_{j, k}^{l}\right)$, respectively, constructed in [23. Theorem 1 and Corollary 2] with respect to $\mathrm{d}_{X \sqcup Y}$ that have the same greatest lower bounds and least upper bounds of $\mathfrak{R e}\left(a_{j, k}^{l}\right), \mathfrak{I m}\left(a_{j, k}^{l}\right)$, respectively.

Now, for every $l \in\{0, \ldots, n\}, j, k \in\left\{0, \ldots, m_{l}\right\}$, define

$$
b_{j, k}^{l}=\left.\widehat{\mathfrak{R e}\left(a_{j, k}^{l}\right)}\right|_{Y}+\left.i \widehat{\mathfrak{I m}\left(a_{j, k}^{l}\right)}\right|_{Y},
$$

where $\left.\right|_{Y}$ denotes restriction to $Y \hookrightarrow X \sqcup Y$. And, note that by construction $b=$ $\left(b_{j, k}^{l}\right)_{l \in\{0, \ldots, n\}, j, k \in\left\{1, \ldots, m_{l}\right\}} \in \mathfrak{s a}(C(Y, \mathfrak{A}))$ and $l_{\left.\mathrm{d}_{Y}, \mathfrak{A}_{\mathbb{R}}\right)}(b) \leqslant 1$.

Next, by the proof of Proposition 4.2 and the fact $\mathbb{R} 1_{C(X, \mathfrak{A})}$ is finite-dimensional and thus satisfies best approximation, there exists $k_{a} \in \mathbb{R}$ such that

$$
\sup _{x \in X}\left\|a(x)-k_{a} 1_{\mathfrak{A}}\right\|_{\infty, \mathfrak{A}_{\mathbb{R}}}=\inf _{r \in \mathbb{R}} \sup _{x \in X}\left\|a(x)-r 1_{\mathfrak{A}}\right\|_{\infty, \mathfrak{A}_{\mathbb{R}}} \leqslant 1
$$

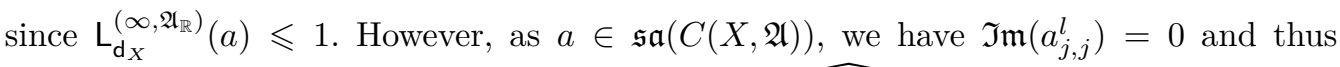
$\mathfrak{I m}\left(b_{j, j}^{l}\right)=0$ by construction, which implies $b_{j, j}^{l}=\left.\widehat{\mathfrak{R e}\left(a_{j, j}^{l}\right)}\right|_{Y}$ for all $l \in\{0, \ldots, n\}$ and $j \in\left\{1, \ldots, m_{l}\right\}$. Therefore, if $z \in Y$, then by 4.7) and preservation of greatest lower bounds and least upper bounds of McShane's extensions

$$
-1 \leqslant \inf _{y \in Y}\left\{\widehat{\mathfrak{R e}\left(a_{j, j}^{l}\right)}(y)-k_{a}\right\} \leqslant b_{j, j}^{l}(z)-k_{a} \leqslant \sup _{y \in Y}\left\{\widehat{\mathfrak{R e}\left(a_{j, j}^{l}\right)}(y)-k_{a}\right\} \leqslant 1,
$$

and so $\sup _{y \in Y}\left|b_{j, j}^{l}(y)-k_{a}\right| \leqslant 1$ for all $l \in\{0, \ldots, n\}$ and $j \in\left\{1, \ldots, m_{l}\right\}$. Similarly, $\sup _{y \in Y}\left|b_{j, k}^{l}(y)\right|_{\infty} \leqslant 1$ for all $l \in\{0, \ldots, n\}$ and $j, k \in\left\{1, \ldots, m_{l}\right\}$. Hence, as $k_{a}$ is only subtracted from diagonal entries, we conclude:

$$
\inf _{r \in \mathbb{R}} \sup _{y \in Y}\left\|b(y)-r 1_{\mathfrak{A}}\right\|_{\infty, \mathfrak{A}_{\mathbb{R}}} \leqslant \sup _{y \in Y}\left\|b(y)-k_{a} 1_{\mathfrak{A}}\right\|_{\infty, \mathfrak{A}_{\mathbb{R}}} \leqslant 1,
$$

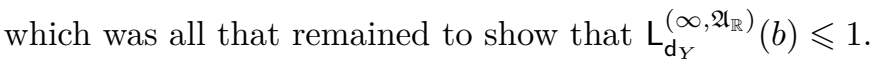

Now, let $(x, y) \in W$. Fix $l \in\{0, \ldots, n\}, j, k \in\left\{1, \ldots, m_{l}\right\}$. We have:

$$
\begin{aligned}
& \left|\left(\pi_{X} \circ a_{j, k}^{l}\right)(x, y)-\left(\pi_{Y} \circ b_{j, k}^{l}\right)(x, y)\right|_{\infty}=\left|a_{j, k}^{l}(x)-b_{j, k}^{l}(y)\right|_{\infty} \\
& \left.\left.\left.\left.=\max \left\{\mid \widehat{\mathfrak{R e}\left(a_{j, k}^{l}\right.}\right)(x)-\widehat{\mathfrak{R e}\left(a_{j, k}^{l}\right.}\right)(y)|,| \widehat{\mathfrak{I m}\left(a_{j, k}^{l}\right.}\right)(x)-\widehat{\mathfrak{I m}\left(a_{j, k}^{l}\right.}\right)(y) \mid\right\} \\
& \leqslant \max \left\{\mathrm{d}_{X \sqcup Y}(x, y), \mathrm{d}_{X \sqcup Y}(x, y)\right\} \leqslant \delta_{X, Y}+\frac{\varepsilon}{2 \sqrt{2} \cdot m_{\mathfrak{A}}} .
\end{aligned}
$$


Hence

$$
\sup _{(x, y) \in W}\left\|\left(\pi_{X}^{\mathfrak{A}} \circ a\right)(x, y)-\left(\pi_{Y}^{\mathfrak{A}} \circ b\right)(x, y)\right\|_{\infty, \mathfrak{A}_{\mathbb{R}}} \leqslant \delta_{X, Y}+\frac{\varepsilon}{2 \sqrt{2} \cdot m_{\mathfrak{A}}},
$$

and therefore, by 4.2 :

$$
\left\|\left(\pi_{X}^{\mathfrak{A}} \circ a\right) 1_{C(W, \mathfrak{A})}-1_{C(W, \mathfrak{A})}\left(\pi_{Y}^{\mathfrak{A}} \circ b\right)(x, y)\right\|_{C(W, \mathfrak{A})} \leqslant \sqrt{2} \cdot m_{\mathfrak{A}} \cdot \delta_{X, Y}+\frac{\varepsilon}{2} .
$$

The argument is symmetric if we began with an element in $b \in \mathfrak{s a}(C(Y, \mathfrak{A}))$ such that

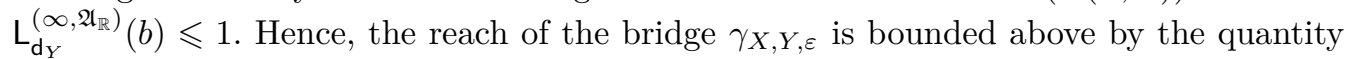
$\sqrt{2} \cdot m_{\mathfrak{A}} \cdot \delta_{X, Y}+\frac{\varepsilon}{2}$ by 20 . Definition 3.14], and thus so is its length. Thus, by TheoremDefinition 1.7, we have

$$
\Lambda\left(\left(C(X, \mathfrak{A}), \mathrm{L}_{\mathrm{d}_{X}}^{\left(\infty, \mathfrak{A}_{\mathbb{R}}\right)}\right),\left(C(Y, \mathfrak{A}), \mathrm{L}_{\mathrm{d}_{Y}}^{\left(\infty, \mathfrak{A}_{\mathbb{R}}\right)}\right)\right) \leqslant \sqrt{2} \cdot m_{\mathfrak{A}} \cdot \delta_{X, Y}+\frac{\varepsilon}{2},
$$

for all $\varepsilon>0$. Thus

$$
\begin{aligned}
\Lambda\left(\left(C(X, \mathfrak{A}), \mathrm{L}_{\mathrm{d}_{X}}^{\left(\infty, \mathfrak{A}_{\mathbb{R}}\right)}\right),\left(C(Y, \mathfrak{A}), \mathrm{L}_{\left.\mathrm{d}_{Y}, \mathfrak{A}_{\mathbb{R}}\right)}^{(\infty}\right)\right. & \leqslant \sqrt{2} \cdot m_{\mathfrak{A}} \cdot \delta_{X, Y} \\
& =\sqrt{2} \cdot m_{\mathfrak{A}} \cdot \mathrm{GH}\left(\left(X, \mathrm{~d}_{X}\right),\left(Y, \mathrm{~d}_{Y}\right)\right)
\end{aligned}
$$

which completes the proof.

As a corollary, we show that we may find finite-dimensional approximations to $C(X, \mathfrak{A})$ when $X$ is compact metric and $\mathfrak{A}$ is finite-dimensional. Thus, we provide many new examples of the propinquity extending the notion of approximate finite-dimensionality since $C(X, \mathfrak{A})$ need not be an AF algebra in general.

Corollary 4.5. Let $\mathfrak{A}$ be a finite-dimensional $C^{*}$-algebra such that there exists $n \in \mathbb{N}$ and $m_{l} \in \mathbb{N} \backslash\{0\}$ for $l \in\{0, \ldots, n\}$ with $\mathfrak{A}=\bigoplus_{l=0}^{n} M_{m_{l}}(\mathbb{C})$. Let $m_{\mathfrak{A}}=\max _{l \in\{0, \ldots, n\}} m_{l}$.

If $\left(X, \mathrm{~d}_{X}\right)$ is a compact metric space, there there exists a sequence $\left(X_{n}\right)_{n \in \mathbb{N}}$ of finite subsets of $X$ such that

$$
\lim _{n \rightarrow \infty} \Lambda\left(\left(C\left(X_{n}, \mathfrak{A}\right), \mathrm{L}_{\mathrm{d}_{X}}^{\left(\infty, \mathfrak{A}_{\mathbb{R}}\right)}\right),\left(C(X, \mathfrak{A}), \mathrm{L}_{\mathrm{d}_{X}}^{\left(\infty, \mathfrak{A}_{\mathbb{R}}\right)}\right)\right)=0 .
$$

In particular, as $C\left(X_{n}, \mathfrak{A}\right)$ is finite-dimensional for all $n \in \mathbb{N}$, there exists a sequence of finite-dimensional $C^{*}$-algebras equipped with $\left(\sqrt{2} \cdot m_{\mathfrak{A}}, 0\right)$-quasi-Leibniz Lip-norms converging in the propinquity to $C(X, \mathfrak{A})$ equipped with a $\left(\sqrt{2} \cdot m_{\mathfrak{A}}, 0\right)$-quasi-Leibniz Lip-norm.

Proof. Since $X$ is compact metric and thus totally bounded, there exists a sequence $\left(X_{n}\right)_{n \in \mathbb{N}}$ of finite subsets of $X$, such that $\lim _{n \rightarrow \infty}$ Haus $_{\mathrm{d}_{X}}\left(X_{n}, X\right)=0$. As the GromovHausdorff distance is bounded above by the Hausdorff distance on compact subsets of a fixed compact metric space, the conclusion follows by Theorem 4.4

Now, on $C(X)$, the classical Lipschitz seminorm $\mathrm{L}_{\mathrm{d}_{X}}$ differs from the Lip-norm $\mathrm{L}_{\mathrm{d}_{X}}^{\left(\infty, \mathbb{C}_{\mathbb{R}}\right)}$ of Notation 4.1 in general due its inclusion of the quotient norm. Thus, our setting does not recover the setting of Theorem 1.9 in general. However, if we allow ourselves to focus on classes of compact metric spaces with a fixed upper bound on diameter and we further provide a slight adjustment to our Lip-norms $\mathrm{L}_{\mathrm{d}_{X}}^{\left(\infty, \mathfrak{A}_{\mathbb{R}}\right)}$ with respect to this bound, then we can recover Theorem 1.9 when $\mathfrak{A}=\mathbb{C}$ by Theorem 4.4 on these particular classes of compact metric spaces. 
TheOrem 4.6. Let $\mathfrak{A}$ be a finite-dimensional $C^{*}$-algebra such that there exists $n \in \mathbb{N}$ and $m_{l} \in \mathbb{N} \backslash\{0\}$ for $l \in\{0, \ldots, n\}$ with $\mathfrak{A}=\bigoplus_{l=0}^{n} M_{m_{l}}(\mathbb{C})$. Let $m_{\mathfrak{A}}=\max _{l \in\{0, \ldots, n\}} m_{l}$.

Fix $K>0$. Denote the class of compact metric spaces with diameter less than or equal to $K$ by $\mathrm{CMS}_{K}$.

For every $\left(X, \mathrm{~d}_{X}\right) \in \mathrm{CMS}_{K}$, if we define for all $a \in \mathfrak{s a}(C(X, \mathfrak{A}))$

$$
\mathrm{L}_{\mathrm{d}_{X}, K}^{\left(\infty, \mathfrak{A}_{\mathbb{R}}\right)}(a)=\max \left\{l_{\mathrm{d}_{X}}^{\left(\infty, \mathfrak{A}_{\mathbb{R}}\right)}(a), \frac{2}{K} \cdot \inf _{r \in \mathbb{R}} \sup _{x \in X}\left\|a(x)-r 1_{\mathfrak{A}}\right\|_{\infty, \mathfrak{A}_{\mathbb{R}}}\right\},
$$

and use Convention 1.8, then:

(1) $\left(C(X, \mathfrak{A}), \mathrm{L}_{\mathrm{d}_{X}, K}^{\left(\infty, \mathfrak{A}_{\mathbb{R}}\right)}\right)$ is a $\left(\sqrt{2} \cdot m_{\mathfrak{A}}, 0\right)$-quasi-Leibniz compact quantum metric space such that $\operatorname{diam}\left(\mathscr{S}(C(X, \mathfrak{A})), \mathrm{mk}_{\mathrm{L}_{\mathrm{d}_{X}, K}^{\left(\infty, \mathfrak{A}_{\mathbb{R}}\right)}}\right) \leqslant K \cdot \sqrt{2} \cdot m_{\mathfrak{A}}$,

(2) the map $\Gamma^{\mathfrak{A}}$ of Theorem 4.4 is well-defined and continuous if $\mathrm{L}_{\mathrm{d}_{X}}^{\left(\infty, \mathfrak{A}_{\mathbb{R}}\right)}$ and CMS are replaced with $\mathrm{L}_{\mathrm{d}_{X}, K}^{\left(\infty, \mathfrak{A}_{\mathbb{R}}\right)}$ and $\mathrm{CMS}_{K}$, and

(3) if $n=0$ and $m_{0}=1$ so that $\mathfrak{A}=\mathbb{C}$, then $\mathrm{L}_{\mathrm{d}_{X}, K}^{\left(\infty, \mathbb{C}_{\mathbb{R}}\right)}=\mathrm{L}_{\mathrm{d}_{X}}$ on $\mathfrak{s a}(C(X))$, and thus the map $\Gamma^{\mathbb{C}}$ of Theorem 4.4 with, $\mathrm{L}_{\mathrm{d}_{X}}^{\left(\infty, \mathbb{C}_{\mathbb{R}}\right)}$ and CMS replaced with $\mathrm{L}_{\mathrm{d}_{X}, K}^{\left(\infty, \mathbb{C}_{\mathbb{R}}\right)}$ and $\mathrm{CMS}_{K}$, is a homeomorphism onto its image.

In particular, if $\mathcal{K}$ is any compact class of compact metric spaces with respect to the Gromov-Hausdorff distance topology, then (1)-(3) are true for $\mathrm{CMS}_{K}$ replaced with $\mathcal{K}$, wherever $\mathrm{CMS}_{K}$ appears and the $K>0$ used for the Lip-norms is any fixed bound on the diameter of all compact metric spaces in $\mathcal{K}$.

Proof. The proof of (1) follows from the methods of Section 2 The proof of (2) follows from the proof of Theorem 4.4 along with the fact that $K$ is fixed. Thus, (3) remains.

We note that $l_{\left.\mathrm{d}_{X}, \mathbb{C}_{\mathbb{R}}\right)}^{\left(\mathrm{L}_{\mathrm{d}}\right.}=\mathrm{L}_{\mathrm{d}_{X}}$ on $\mathfrak{s a}(C(X))$. Thus, to show $\mathrm{L}_{\mathrm{d}_{X}, K}^{\left(\infty, \mathbb{C}_{\mathbb{R}}\right)}=\mathrm{L}_{\mathrm{d}_{X}}$ on $\mathfrak{s a}(C(X))$, we only need to verify that $\frac{2}{K} \cdot \inf _{r \in \mathbb{R}} \sup _{x \in X}\left\|f(x)-r 1_{\mathbb{C}}\right\|_{\infty, \mathbb{C}_{\mathbb{R}}} \leqslant \mathrm{L}_{\mathrm{d}_{X}}(f)$ for all $f \in \mathfrak{s a}(C(X))$. However, in this setting, the quantity $\inf _{r \in \mathbb{R}} \sup _{x \in X}\left\|(\cdot)(x)-r 1_{\mathbb{C}}\right\|_{\infty, \mathbb{C}_{\mathbb{R}}}$ on $\mathfrak{s a}(C(X))$ is simply the quotient norm with respect $\mathbb{C} 1_{C(X)}$ and the supremum norm with respect to absolute value. Therefore, for all $f \in \mathfrak{s a}(C(X))$, we have:

$$
\frac{2}{K} \cdot \inf _{r \in \mathbb{R}} \sup _{x \in X}\left\|f(x)-r 1_{\mathbb{C}}\right\|_{\infty, \mathbb{C}_{\mathbb{R}}}=\frac{2}{K} \cdot \frac{\left|f\left(x_{m}\right)-f\left(x_{M}\right)\right|}{2},
$$

where $x_{m}$ achieves the minimum of $f$ on $X$ and $x_{M}$ achieves the maximum of $f$ on $X$. Now, if $\operatorname{diam}\left(X, \mathrm{~d}_{X}\right)=0$, then $C(X)=\mathbb{C}$, and so any Lip-norm is the 0-seminorm on $\mathfrak{s a}(C(X))$, which completes this case. So, for the remainder of the proof, we assume that $\operatorname{diam}\left(X, \mathrm{~d}_{X}\right)>0$. Thus

$$
\begin{aligned}
\frac{2}{K} \cdot \frac{\left|f\left(x_{m}\right)-f\left(x_{M}\right)\right|}{2} \leqslant \sup _{x, y \in X}\left\{\frac{|f(x)-f(y)|}{K}\right\} \leqslant \sup _{x, y \in X}\left\{\frac{|f(x)-f(y)|}{\operatorname{diam}\left(X, \mathrm{~d}_{X}\right)}\right\} \\
=\sup _{x, y \in X, x \neq y}\left\{\frac{|f(x)-f(y)|}{\operatorname{diam}\left(X, \mathrm{~d}_{X}\right)}\right\} \leqslant \sup _{x, y \in X, x \neq y}\left\{\frac{|f(x)-f(y)|}{\mathrm{d}_{X}(x, y)}\right\}=\mathrm{L}_{\mathrm{d}_{X}}(f) .
\end{aligned}
$$

Thus, it must be the case that $\frac{2}{K} \cdot \inf _{r \in \mathbb{R}} \sup _{x \in X}\left\|f(x)-r 1_{\mathbb{C}}\right\|_{\infty, \mathbb{C}_{\mathbb{R}}} \leqslant \mathrm{L}_{\mathrm{d}_{X}}(f)$ by 4.9 , which completes the proof of (3). 
For the remaining statement, we simply note that any compact class of compact metric spaces with respect to the Gromov-Hausdorff distance topology is a subclass of $\mathrm{CMS}_{K}$ for come $K>0$ by the Gromov Compactness Theorem [7, Theorem 7.4.15].

Remark 4.7. Similar conclusions to Proposition 2.14 and the results of Section 3 can be made with respect to the Lip-norm $\mathrm{L}_{\mathrm{d}_{X}, K}^{\left(\infty, \mathfrak{A}_{\mathbb{R}}\right)}$ of Theorem 4.6 , but we do not need them here and do not list them for the purpose of presentation.

Furthermore, we note that (1) and (3) of Theorem 4.6 would still be true if $K$ were replaced by $\operatorname{diam}\left(X, \mathrm{~d}_{X}\right)$. However, for $(2)$, the $K$ is used in a non-trivial yet subtle way since we must compare these spaces in the propinquity and if $K$ were allowed to vary then the method used involving expression (4.8) would fail. And, the importance of (2) is to show that we truly are extending the continuity of the map $\Gamma$ of Theorem 1.9 to the matricial case on the subclass $\mathrm{CMS}_{K}$.

Acknowledgments. The first author was partially supported by the Independent Research Fund Denmark through the project "Classical and Quantum Distances" (grant no. 9040-00107B) and by the Simons Foundation grant 346300 and the Polish Government MNiSW 2015-2019 matching fund, and this work is part of the project supported by the grant H2020-MSCA-RISE-2015-691246-QUANTUM DYNAMICS and the Polish Government grant 3542/H2020/2016/2.

Some of this work was completed during the Simons semester hosted at IMPAN during September-December 2016 titled Noncommutative Geometry - The Next Generation.

\section{References.}

[1] K. Aguilar, AF algebras in the quantum Gromov-Hausdorff propinquity space, submitted, 2016, ArXiv:1612.02404

[2] K. Aguilar, Convergence of quotients of AF algebras in quantum propinquity by convergence of ideals, submitted, 2016, ArXiv:1608.07016.

[3] K. Aguilar, F. Latrémolière, Quantum ultrametrics on AF algebras and the Gromov-Hausdorff propinquity, Studia Math. 231 (2015), 149-194.

[4] B. Blackadar, Operator Algebras. Theory of $C^{*}$-algebras and von Neumann algebras, Encyclopaedia Math. Sci. 122, Operator Algebras and Non-commutative Geometry, III, Springer, Berlin, 2006.

[5] N. Bourbaki, General Topology. Chapters 5-10, Elem. Math. (Berlin), Springer, Berlin, 1998.

[6] N. P. Brown, N. Ozawa, $C^{*}$-algebras and finite-dimensional approximations, Grad. Stud. Math. 88, Amer. Math. Soc., Providence, 2008.

[7] D. Burago, Y. Burago, S. Ivanov, A Course in Metric Geometry, Grad. Stud. Math. 33, Amer. Math. Soc., Providence, 2001.

[8] A. Connes, Compact metric spaces, Fredholm modules and hyperfiniteness, Ergodic Theory Dynam. Systems 9 (1989), 207-220.

[9] A. Connes, Noncommutative Geometry, Academic Press, San Diego, 1994.

[10] J. B. Conway, A Course in Functional Analysis, Grad. Texts in Math. 96, Springer, New York, 1990.

[11] K. R. Davidson, $C^{*}$-algebras by Example, Fields Inst. Monogr., Amer. Math. Soc., Providence, 1996.

[12] R. M. Dudley, Real Analysis and Probability, Cambridge Stud. Adv. Math. 74, Cambridge Univ. Press, Cambridge, 2002. 
[13] G. Elliott, D. Evans, Structure of the irrational rotation $C^{*}$-algebras, Ann. of Math. (2) 138 (1993), 477-501.

[14] R. A. Horn, C. R. Johnson, Matrix Analysis, Cambridge Univ. Press, Cambridge, 1990.

[15] I. Kaplansky, The structure of certain operator algebras, Trans. Amer. Math. Soc. 70 (1951), 219-255.

[16] F. Latrémolière, Bounded-Lipschitz distances on the state space of a $C^{*}$-algebra, Taiwanese J. Math. 11 (2007), 447-469.

[17] F. Latrémolière, Convergence of fuzzy tori and quantum tori for the quantum GromovHausdorff propinquity: an explicit approach, Münster J. Math. 8 (2015), 57-98.

[18] F. Latrémolière, The dual Gromov-Hausdorff propinquity, J. Math. Pures Appl. 103 (2015), 303-351.

[19] F. Latrémolière, Equivalence of quantum metrics with a common domain, J. Math. Anal. Appl. 443 (2016), 1179-1195.

[20] F. Latrémolière, The quantum Gromov-Hausdorff propinquity, Trans. Amer. Math. Soc. 368 (2016), 365-411.

[21] F. Latrémolière, A compactness theorem for the dual Gromov-Hausdorff propinquity, Indiana Univ. Math. J. 66 (2017), 1707-1753.

[22] F. Latrémolière, The modular Gromov-Hausdorff propinquity, Dissertationes Math. 544 (2019).

[23] E. J. McShane, Extension of range of functions, Bull. Amer. Math. Soc. 40 (1934), 825-920.

[24] J. R. Munkres, Topology, second ed., Prentice Hall, Upper Saddle River, 2000.

[25] G. J. Murphy, $C^{*}$-algebras and Operator Theory, Academic Press, Boston, 1990.

[26] N. Ozawa, M. A. Rieffel, Hyperbolic group $C^{*}$-algebras and free product $C^{*}$-algebras as compact quantum metric spaces, Canad. J. Math. 57 (2005), 1056-1079.

[27] M. A. Rieffel, Metrics on states from actions of compact groups, Doc. Math. 3 (1998), 215-229, ArXiv:math.OA/9807084

[28] M. A. Rieffel, Metrics on state spaces, Doc. Math. 4 (1999), 559-600, ArXiv: math.OA/9906151

[29] M. A. Rieffel, Gromov-Hausdorff distance for quantum metric spaces, Mem. Amer. Math. Soc. 168 (2004), no. 796, 1-65, ArXiv:math.OA/0011063

[30] M. A. Rieffel, Compact quantum metric spaces, in: Operator Algebras, Quantization, and Noncommutative Geometry, Contemp. Math. 365, Amer. Math. Soc., Providence, 2005, 315-330.

[31] M. A. Rieffel, Leibniz seminorms and best approximation from $C^{*}$-subalgebras, Sci. China Math. 54 (2011), 2259-2274.

[32] M. A. Rieffel, Matricial bridges for "matrix algebras converge to the sphere", in: Operator Algebras and their Applications, Contemp. Math. 671, Amer. Math. Soc., Providence, RI, 2016, 209-233.

[33] W. Rudin, Functional Analysis, second ed., Internat. Ser. Pure Appl. Math., McGraw-Hill, New York, 1991.

[34] N. Weaver, Lipschitz Algebras, World Scientific, River Edge, NJ, 1999.

[35] S. Willard, General Topology, Dover Publ., Minneola, NY, 2004. 